This item was submitted to Loughborough's Research Repository by the author.

Items in Figshare are protected by copyright, with all rights reserved, unless otherwise indicated.

\title{
Universal constraints on the location of extrema of eigenfunctions of non- local Schrödinger operators
}

PLEASE CITE THE PUBLISHED VERSION

https://doi.org/10.1016/j.jde.2019.01.007

\section{PUBLISHER}

(C) Elsevier

\section{VERSION}

AM (Accepted Manuscript)

\section{PUBLISHER STATEMENT}

This paper was accepted for publication in the journal Journal of Differential Equations and the definitive published version is available at https://doi.org/10.1016/j.jde.2019.01.007.

\section{LICENCE}

CC BY-NC-ND 4.0

\section{REPOSITORY RECORD}

Biswas, Anup, and Jozsef Lorinczi. 2019. "Universal Constraints on the Location of Extrema of Eigenfunctions of Non-local Schrödinger Operators”. figshare. https://hdl.handle.net/2134/27630. 


\title{
UNIVERSAL CONSTRAINTS ON THE LOCATION OF EXTREMA OF EIGENFUNCTIONS OF NON-LOCAL SCHRÖDINGER OPERATORS
}

\author{
ANUP BISWAS AND JÓZSEF LŐRINCZI
}

\begin{abstract}
We derive a lower bound on the location of global extrema of eigenfunctions for a large class of non-local Schrödinger operators in convex domains under Dirichlet exterior conditions, featuring the symbol of the kinetic term, the strength of the potential, and the corresponding eigenvalue, and involving a new universal constant. We show a number of probabilistic and spectral geometric implications, and derive a Faber-Krahn type inequality for non-local operators. Our study also extends to potentials with compact support, and we establish bounds on the location of extrema relative to the boundary edge of the support or level sets around minima of the potential.
\end{abstract}

Declarations of Interest: none

\section{Introduction}

Recently, in the paper [61] the remarkable bound

$$
\operatorname{dist}\left(x^{*}, \partial \mathcal{D}\right) \geq c\left\|\frac{\Delta \varphi}{\varphi}\right\|_{L^{\infty}(\mathcal{D})}^{-1 / 2}
$$

has been obtained on the distance between the location of an assumed global maximum $x^{*}$ of any eigenfunction $\varphi$ of the Schrödinger operator $H=-\Delta+V$ with Dirichlet boundary condition set for a simply connected domain $\mathcal{D} \subset \mathbb{R}^{2}$, and the boundary of $\mathcal{D}$. Here the potential $V$ is bounded and includes the eigenvalue corresponding to $\varphi$, and $c>0$ is a constant, independent of $\mathcal{D}, \varphi$ and $V$. The paper [8] established a similar relationship for the fractional Schrödinger operator $(-\Delta)^{\alpha / 2}+V$, $0<\alpha<2$, for arbitrary dimensions $d \geq 2$, and pointed out some interesting corollaries.

In this paper we consider the problem of the location of extrema in a substantially amplified context and set of goals. While we make use of an inspiring basic idea leading to (1.1), we see it worthwhile to be developed to a far greater extent than attempted by the authors in [61], in order to serve as the beginning of a programme of studying important aspects of local behaviour for a whole class of equations of pure and applied interest. Specifically, our framework is the class of non-local Schrödinger operators of the form

$$
H=\Psi(-\Delta)+V
$$

where $\Psi$ is a so-called Bernstein function, and $V$ is a multiplication operator called potential (for details see Section 2). Such operators have been considered from a combined perturbation theory and functional integration point of view in [35, 36, 41], and we will discuss some motivations below. When $\Psi$ is the identity function, we get back to classical Schrödinger operators, thus this framework also allows comparison with other operators and related equations, and new light is shed also on classical Laplacians with or without potentials.

Anup Biswas, Department of Mathematics, Indian Institute of Science Education and Research, Dr. Homi Bhabha Road, Pune 411008, India, Anup@isserpune.ac.in

József Lőrinczi, Department of Mathematical Sciences, Loughborough University, Loughborough LE11 3TU, United KIngdom, J.Lorinczi@LBoro.AC.UK

2000 Mathematics Subject Classification. 35S15, 47A75, 60G51, $60 \mathrm{~J} 75$.

Key words and phrases. Non-local Schrödinger operators, Bernstein functions, subordinate Brownian motion, Dirichlet exterior value problem, principal eigenvalues and eigenfunctions, hot spots, Faber-Krahn inequality, potential wells. 
We will be interested in the properties of solutions of two eigenvalue problems. The first is a non-local Dirichlet-Schrödinger problem for a bounded convex domain $\mathcal{D} \subset \mathbb{R}^{d}, d \geq 1$, given by

$$
\left\{\begin{array}{cl}
H \varphi=\lambda \varphi & \text { in } \mathcal{D} \\
\varphi=0 & \text { in } \mathcal{D}^{c}
\end{array}\right.
$$

in weak sense. In this case there is a countable set of eigenvalues

$$
\lambda_{1}^{\mathcal{D}, V}<\lambda_{2}^{\mathcal{D}, V} \leq \lambda_{3}^{\mathcal{D}, V} \leq \ldots
$$

of finite multiplicities each, and a corresponding orthonormal set of eigenfunctions $\varphi_{1}, \varphi_{2}, \ldots \in$ $\operatorname{Dom}(H) \subset L^{2}(\mathcal{D})$. When $V \equiv 0$, the problem reduces to the non-local Dirichlet eigenvalue equation. In this case the spectrum is still discrete, and we use the notation $\lambda_{1}^{\mathcal{D}}, \lambda_{2}^{\mathcal{D}}, \ldots$ for the eigenvalues.

The second problem we consider is the eigenvalue equation in $L^{2}\left(\mathbb{R}^{d}\right), d \geq 1$,

$$
H \varphi=\lambda \varphi, \quad \operatorname{supp} V=\mathcal{K}, \text { with } \mathcal{K} \subset \mathbb{R}^{d} \text { bounded. }
$$

In particular, this covers potential wells of depth $v>0$, when $V=-v \mathbb{1}_{\mathcal{K}}$, which are of basic interest. In this case appropriate conditions will be needed on $V$ in order to have any $L^{2}$-eigenfunctions. The Dirichlet-Schrödinger problem can also be seen as a Schrödinger problem in full space, where the potential equals $V$ in $\mathcal{D}$ and infinity elsewhere.

Non-local (i.e., integro-differential) equations are gaining increasing interest recently from the corners of both pure and applied mathematics. While PDE based on the Laplacian and related elliptic operators proved to be ubiquitous in virtually every fundamental model of dynamics for a long time, it is now recognized that a new range of effects is captured if one uses a class of non-local operators, in which the classical Laplacian is just one special case. Much work has been done recently on the well-posedness and regularity theory of such equations, see, e.g., [20, 62] and many related references. There is also much interest due to the fact that non-local operators are generators of Lévy or Feller processes [16, 38], and their study is made possible by probabilistic and potential theory methods. On the other hand, applications to mathematical physics, such as anomalous transport [46, 58] and quantum theory [25, 37, 53], or more computationally, image reconstruction via denoising $[18,29]$, to name just a few, provide a continuing incentive to the development of these ideas and techniques.

The study of non-local Schrödinger equations is one aspect of this work, and some primary work on developing a related potential theory has been done in $[11,12]$. There are many possible choices of $\Psi$ of interest in applications. The fractional Laplacian $\Psi(-\Delta)=(-\Delta)^{\alpha / 2}, 0<\alpha<2$, is the most studied of them. There is a range of exponents $\alpha$ used in anomalous transport theory, however, there are many further applications, e.g., $\alpha=1.3$ describes the dynamics of particles trapped in the vortices of a flow, $\alpha=1.5$ relates with the spatial distribution of the gravitational field generated by a cluster of uniformly distributed stars, etc. The relativistic Laplacians $\Psi(-\Delta)=\left(-\Delta+m^{2 / \alpha}\right)^{\alpha / 2}-$ $m, m>0$, are used to describe relativistic or photonic quantum effects, geometric stable operators $\Psi(-\Delta)=\log \left(1+(-\Delta)^{\alpha / 2}\right), 0<\alpha \leq 2$, are more used in studying financial processes [60], and so on. For a more detailed discussion we refer to [43]. We also note that qualitatively different spectral and analytic behaviours of $H$ and the related semigroup occur in function of the choice of $\Psi$. For instance, for operators and related processes for which the singular integral (Lévy jump) kernel is polynomially or sub-exponentially decaying (e.g., fractional Laplacian), the eigenfunctions have very different asymptotic behaviours than for exponentially or super-exponentially decaying kernels (e.g., relativistic Laplacian), and a phase transition-like phenomenon occurs (for details see [44, Sect. 4.4]). All this shows that these operators, in diverse aspects, massively differ from the classical Laplacian, and also produce spectacular differences between themselves.

Explicit solutions of eigenvalue problems for non-local Schrödinger operators are rare. In [56] this has been obtained for the operator $\left(-d^{2} / d x^{2}\right)^{1 / 2}+x^{2}$, and in [27] for $\left(-d^{2} / d x^{2}\right)^{1 / 2}+x^{4}$, both in $L^{2}(\mathbb{R})$. A detailed study of the asymptotic behaviour at infinity of the eigenfunctions for a large 
class of non-local Schrödinger operators has been made in [42, 44]. Bounds, monotonicity and continuity properties for Dirichlet eigenvalues for large classes of domains have been established in $[23,24]$, approximate solutions and detailed estimates for some non-local Dirichlet problems in intervals, half-spaces or boxes have been presented in $[40,41,49,50,51]$. Since an explicit computation of the principal Dirichlet eigenvalue or eigenfunction is not available even for the simplest cases, a study of the properties of the spectrum becomes important. Some results on the shape of eigenfunctions or solutions were obtained in [4, 48], and [57] investigates the local behaviour of eigenfunctions for potentials wells.

Our results in this paper contribute to a study of local properties of eigenfunctions of non-local Schrödinger operators. There are several reasons why information on the location of extrema of eigenfunctions is of interest, and we single out a few here as follows:

(i) Maximum principles: Maximum principles are fundamental tools in the study of elliptic and parabolic linear, and semilinear problems. Using our present work and the techniques developed here for non-local operators, we are able to derive and prove elliptic and parabolic Aleksandrov-Bakelman-Pucci type estimates, Berestycki-Nirenberg-Varadhan type refined maximum principles, anti-maximum principles in the sense of Clément-Peletier, a maximum principle for narrow domains, as well as Liouville theorems. This is presented in detail elsewhere, see [9], and for the context of time-fractional evolutions [10]. We note that using our techniques all this could be implemented in the framework of viscosity solutions.

(ii) Hot-spots: A hot-spot is a point in space where the solution of the heat equation in a bounded domain at a given time attains its maximum, and an object of study for classical domain Laplacians has been how they move in time when Neumann or Dirichlet boundary conditions are imposed. For Dirichlet boundary conditions, on the long run the solution increasingly takes the shape of the principal eigenfunction, and the hot-spot becomes its maximizer. While there are several classical results on this challenging problem, we mention [31], in which the problem is studied for bounded convex sets in $\mathbb{R}^{2}$, and the recent paper [17] which obtained a lower bound on the location of the maximum of the principal Dirichlet eigenfunction. One implication of our results is a significantly improved bound, see a discussion in Remark 3.7 and Corollary 3.4 below.

(iii) Torsion: The torsion function is the solution of a specific Dirichlet boundary value problem, with interest originally derived from mechanics and also having an important probabilistic meaning. A puzzling phenomenon is that its maximizer and the maximizer of the principal Dirichlet eigenfunction of the Laplacian are located very near to each other, though they fail to coincide, see $[7,34]$ and the references therein. In our present work we also obtain a result on this for the non-local case, for a further discussion see Remark 3.9.

(iv) Most likely location of paths: Since the principal eigenfunction of $H$ can be chosen to be strictly positive, by its harmonicity it can be used as a Doob $h$-transform to construct a stochastic process obtained under the perturbation of $V$ of the subordinate Brownian motion generated by $\Psi(-\Delta)$. In case of a classical Schrödinger operator this is a diffusion, while for non-local cases it is a Lévy-type jump process with, in general, unbounded coefficients. In both cases the maximizer of the first eigenfunction gives the mode of the stationary probability density of this process, i.e., describes the location in space which gives the highest contribution into the distribution of paths. This is discussed in further detail in Remark 3.4 below.

(v) An application to modelling groundwater contamination: An application of high practical interest of anomalous transport described by non-local equations is a more realistic description of the spread of contaminated groundwater by taking into account non-uniformities of a porous soil, see [45] and references therein. The maximizer(s) in this case have a relevance in the localization of the highest-concentration points of the plume. Also, in this context the study of inverse problems become important in order to control the level sets 
and maxima of the plume. Using our techniques we have been able to discuss an inverse source problem in [10, Th. 3.6], which is just the beginning of a series of investigations of a practical relevance.

To conclude, we outline the main results and highlight some technical achievements in this paper, apart from what we discussed above.

(1) Our key results are stated in Theorems 3.1-3.2, and we will study their multiple implications involving an interplay of probabilistic and spectral geometric aspects. Our results reproduce (1.1) as a specific case, however, apart from a far more general framework our work here goes well beyond [61] on several counts. One is that our expressions feature the symbol of the kinetic part of the operator. This allows us to understand what is behind the formulae involving a lower bound on the position of extrema, and it will turn out from the probabilistic representation that it results from a balance of two survival times of paths of the related random process running in the domain (Remark 3.4). This points to an underlying mechanism fundamentally involving the competition of energy versus entropy effects, and offers a very different perspective. A second point how we reach a different level of discussion is that we allow a large class of potentials, including local singularities, and do not limit ourselves to bounded potentials. This has not been attempted in [61], and controlling such a possibly very "rugged" potential landscape is not a straightforward step from bounded potentials. We also note that our combined analytic and probabilistic techniques developed here allow to cover general convex domains (see Theorem 3.1 and Remark 3.1), removing boundary regularity problems often encountered when using purely analytic means. This will also be helpful when considering maximum principles in [9].

(2) Apart from bounded domains we also consider potentials with compact support in full space $\mathbb{R}^{d}$, and derive predictions on the location of extrema relative to the edge of their supports or from neighbourhoods (e.g., level sets) of the minima of the potential, see Section 4 and specifically the key Theorems 4.2 and 4.5 below. There is very little information on this even for the classical and, as far as we are aware, nothing for non-local Schrödinger operators. We also note that this problem has not been addressed in [61]. As consequences, we observe some interesting behaviours dependent on whether the potential is attracting or repelling (Theorem 4.4).

(3) As it will be seen in what follows, the localization of the extrema of Dirichlet-Schrödinger eigenfunctions is, roughly speaking, an isoperimetric-type property, determined by underlying geometric principles. In Corollary 3.5 we obtain a new Faber-Krahn type inequality for non-local Schrödinger operators as a direct consequence of the estimates on the location of extrema.

(4) We also obtain a variety of geometric and probabilistic bounds on the eigenvalues in the spirit of the discussions in $[3,5]$ and references therein. In particular, we derive a lower estimate on all moments of exit times of subordinate Brownian motion from convex domains, and further relations on eigenvalues (Corollaries 3.2 and 3.3).

The remainder of this paper is organized as follows. In Section 2 we discuss some properties of Bernstein functions $\Psi$ on which we rely throughout below when using the operators $\Psi(-\Delta)$ and related subordinate Brownian motions. In Section 3 first we establish some basic facts on the Dirichlet-Schrödinger eigenvalue problem, which do not seem to be available in the literature. Next we state and prove our main results in Theorems 3.1-3.2, and then discuss a number of consequences and implications in corollaries and a string of remarks. Section 4 is devoted to operators having potentials with compact support. 


\section{Bernstein functions of the Laplacian and subordinate Brownian motions}

Now we turn to describe the above objects formally. Denote

$$
H_{0}=\Psi(-\Delta),
$$

where $\Psi$ is a Bernstein function given below. This operator can be defined via functional calculus by using the spectral decomposition of the Laplacian. It is a pseudo-differential operator whose symbol is given by the Fourier multiplier

$$
\widehat{H_{0} f}(y)=\Psi\left(|y|^{2}\right) \widehat{f}(y), \quad y \in \mathbb{R}^{d}, f \in \operatorname{Dom}\left(H_{0}\right),
$$

with domain $\operatorname{Dom}\left(H_{0}\right)=\left\{f \in L^{2}\left(\mathbb{R}^{d}\right): \Psi\left(|\cdot|^{2}\right) \widehat{f} \in L^{2}\left(\mathbb{R}^{d}\right)\right\}$. It follows by general arguments that $H_{0}$ is a positive, self-adjoint operator with core $C_{\mathrm{c}}^{\infty}\left(\mathbb{R}^{d}\right)$, for details see [36, 64].

Recall that a Bernstein function is a non-negative completely monotone function, i.e., an element of the set

$$
\mathcal{B}=\left\{f \in C^{\infty}((0, \infty)): f \geq 0 \text { and }(-1)^{n} \frac{d^{n} f}{d x^{n}} \leq 0, \text { for all } n \in \mathbb{N}\right\} .
$$

In particular, Bernstein functions are increasing and concave. We will make use below of the subset

$$
\mathcal{B}_{0}=\left\{f \in \mathcal{B}: \lim _{u \downarrow 0} f(u)=0\right\} .
$$

Let $\mathcal{M}$ be the set of Borel measures $\mu$ on $\mathbb{R} \backslash\{0\}$ with the property that

$$
\mu((-\infty, 0))=0 \quad \text { and } \quad \int_{\mathbb{R} \backslash\{0\}}(y \wedge 1) \mu(d y)<\infty .
$$

Notice that, in particular, $\int_{\mathbb{R} \backslash\{0\}}\left(y^{2} \wedge 1\right) \mu(d y)<\infty$ holds, thus $\mu$ is a Lévy measure supported on the positive semi-axis. It is well-known then that every Bernstein function $\Psi \in \mathcal{B}_{0}$ can be represented in the form

$$
\Psi(u)=b u+\int_{(0, \infty)}\left(1-e^{-y u}\right) \mu(\mathrm{d} y)
$$

with $b \geq 0$, moreover, the map $[0, \infty) \times \mathcal{M} \ni(b, \mu) \mapsto \Psi \in \mathcal{B}_{0}$ is bijective. $\Psi$ is said to be a complete Bernstein function if there exists a Bernstein function $\widetilde{\Psi}$ such that

$$
\Psi(u)=u^{2} \mathcal{L}(\widetilde{\Psi})(u), \quad u>0,
$$

where $\mathcal{L}$ stands for Laplace transform. It is known that every complete Bernstein function is also a Bernstein function. Also, for a complete Bernstein function the Lévy measure $\mu(\mathrm{d} y)$ has a completely monotone density with respect to the Lebesgue measure. The class of complete Bernstein functions is large, including important cases such as

(i) $\Psi(u)=u^{\alpha / 2}, \alpha \in(0,2]$

(ii) $\Psi(u)=\left(u+m^{2 / \alpha}\right)^{\alpha / 2}-m, m \geq 0, \alpha \in(0,2)$

(iii) $\Psi(u)=u^{\alpha / 2}+u^{\beta / 2}, 0<\beta<\alpha \in(0,2]$

(iv) $\Psi(u)=\log \left(1+u^{\alpha / 2}\right), \alpha \in(0,2]$

(v) $\Psi(u)=u^{\alpha / 2}(\log (1+u))^{\beta / 2}, \alpha \in(0,2), \beta \in(0,2-\alpha)$

(vi) $\Psi(u)=u^{\alpha / 2}(\log (1+u))^{-\beta / 2}, \alpha \in(0,2], \beta \in[0, \alpha)$.

On the other hand, the Bernstein function $\Psi(u)=1-e^{-u}$ is not a complete Bernstein function. For a detailed discussion we refer to the monograph [64].

Bernstein functions are closely related to subordinators, and we will use this relationship below. Recall that a one-dimensional Lévy process $\left(S_{t}\right)_{t \geq 0}$ on a probability space $\left(\Omega_{S}, \mathcal{F}_{S}, \mathbb{P}_{S}\right)$ is called a subordinator whenever it satisfies $S_{s} \leq S_{t}$ for $s \leq t, \mathbb{P}_{S}$-almost surely. A basic fact is that the Laplace transform of a subordinator is given by a Bernstein function, i.e.,

$$
\mathcal{L}\left(S_{t}\right)(u)=\mathbb{E}_{\mathbb{P}_{S}}\left[e^{-u S_{t}}\right]=e^{-t \Psi(u)}, \quad t \geq 0,
$$


holds, where $\Psi \in \mathcal{B}_{0}$. In particular, there is a bijection between the set of subordinators on a given probability space and Bernstein functions with vanishing right limits at zero; to emphasize this, we will occasionally write $\left(S_{t}^{\Psi}\right)_{t \geq 0}$ for the unique subordinator associated with Bernstein function $\Psi$. Corresponding to the examples above, the related processes are (i) $\alpha / 2$-stable subordinator, (ii) relativistic $\alpha / 2$-stable subordinator, (iii) sums of independent subordinators of different indeces, (iv) geometric $\alpha / 2$-stable subordinators (specifically, the Gamma-subordinator for $\alpha=2$ ), etc. The non-complete Bernstein function mentioned above describes the Poisson subordinator.

Let $\left(B_{t}\right)_{t>0}$ be $\mathbb{R}^{d}$-valued a Brownian motion on Wiener space $\left(\Omega_{W}, \mathcal{F}_{W}, \mathbb{P}_{W}\right)$, running twice as fast as standard $d$-dimensional Brownian motion, and let $\left(S_{t}^{\Psi}\right)_{t \geq 0}$ be an independent subordinator. The random process

$$
\Omega_{W} \times \Omega_{S} \ni(\omega, \varpi) \mapsto B_{S_{t}(\varpi)}(\omega) \in \mathbb{R}^{d}
$$

is called subordinate Brownian motion under $\left(S_{t}^{\Psi}\right)_{t \geq 0}$. For simplicity, we will denote a subordinate Brownian motion by $\left(X_{t}\right)_{t \geq 0}$, its probability measure for the process starting at $x \in \mathbb{R}^{d}$ by $\mathbb{P}^{x}$, and expectation with respect to this measure by $\mathbb{E}^{x}$. Every subordinate Brownian motion is a Lévy process, with infinitesimal generator $H_{0}=\Psi(-\Delta)$. Subordination then gives the expression

$$
\mathbb{P}\left(X_{t} \in E\right)=\int_{0}^{\infty} \mathbb{P}_{W}\left(B_{s} \in E\right) \mathbb{P}_{S}\left(S_{t} \in \mathrm{d} s\right),
$$

for every measurable set $E$.

Our main concern in what follows are some properties in the bulk of functions satisfying the eigenvalue equations (1.3-1.4) in weak sense. Specifically, we will focus on the location of extrema of eigenfunctions by using a stochastic representation of the solutions, featuring subordinate Brownian motion.

\section{Constraints on the location of extrema}

\subsection{The Dirichlet-Schrödinger problem}

In this section we assume $\mathcal{D} \subset \mathbb{R}^{d}$ to be a bounded open set. Consider a complete Bernstein function $\Psi$ and the operator $H_{0}=\Psi(-\Delta)$ on $L^{2}\left(\mathbb{R}^{d}\right)$. The Dirichlet eigenvalue problem (1.3) for $V \equiv 0$ has been studied in various papers, including [23, 24, 41, 50, 51]. In particular, the following holds; for details we refer to [41] and [28]. Consider the space $C_{\mathrm{c}}^{\infty}(\mathcal{D})$, and define the operator $H_{0}^{\mathcal{D}}$ given by the Friedrichs extension of $\left.H_{0}\right|_{C_{\mathrm{c}}^{\infty}(\mathcal{D})}$. It can be shown that the form-domain of $H_{0}^{\mathcal{D}}$ contains those functions that are in the form-domain of $H_{0}$ and are almost surely zero outside of $\mathcal{D}$. Furthermore, the operator $-H_{0}^{\mathcal{D}}$ generates the strongly continuous operator semigroup

$$
T_{t}^{\mathcal{D}}=e^{-t H_{0}^{\mathcal{D}}}, \quad t \geq 0
$$

Each operator $T_{t}^{\mathcal{D}}$ is a contraction on $L^{p}(\mathcal{D})$, for every $p \geq 1$, including $p=\infty$. When $\Psi$ is unbounded, $T_{t}^{\mathcal{D}}$ is a contraction also on $C_{0}(\mathcal{D})$. If $e^{-t \Psi\left(|x|^{2}\right)} \in L^{1}(\mathcal{D})$ for $t>0$, then each $T_{t}^{\mathcal{D}}$ is a Hilbert-Schmidt operator, in particular, they are compact. Hence, by general theory, the equation

$$
T_{t}^{\mathcal{D}} \varphi=e^{-\lambda t} \varphi, \quad t>0,
$$

is solved by a countable set of eigenvalues $\lambda_{1}^{\mathcal{D}}<\lambda_{2}^{\mathcal{D}} \leq \lambda_{3}^{\mathcal{D}} \leq \cdots \rightarrow \infty$, of finite multiplicity each, corresponding to an orthonormal set of eigenfunctions $\varphi_{1}^{\mathcal{D}}, \varphi_{2}^{\mathcal{D}}, \ldots \in L^{2}(\mathcal{D})$. The principal eigenvalue $\lambda_{1}^{\mathcal{D}}$ has multiplicity one, and the principal eigenfunction $\varphi_{1}^{\mathcal{D}}$ has a strictly positive version, which we will adopt throughout. Moreover, due to strong continuity of the semigroup, the spectrum is independent of $t>0$, in particular, since $-H_{0}^{\mathcal{D}}$ is the infinitesimal generator of $\left\{T_{t}^{\mathcal{D}}: t \geq 0\right\}$, the same eigenvalues and eigenfunctions also solve $(1.3)$ for $V \equiv 0$. It is also known that $\left\{T_{t}^{\bar{D}}: t \geq 0\right\}$ is the Markov semigroup of killed subordinate Brownian motion, i.e., we have

$$
T_{t}^{\mathcal{D}} f(x)=\mathbb{E}^{x}\left[f\left(X_{t}\right) \mathbb{1}_{\left\{\tau_{D}>t\right\}}\right], \quad x \in \mathcal{D}, t>0, f \in L^{2}(\mathcal{D}),
$$


where

$$
\tau_{\mathcal{D}}=\inf \left\{t>0: X_{t} \notin \mathcal{D}\right\}
$$

is the first exit time of $\left(X_{t}\right)_{t \geq 0}$ from $\mathcal{D}$.

In contrast to the pure Dirichlet problem, the Dirichlet-Schrödinger problem (1.3) with $V \not \equiv 0$ has been much less studied and the counterparts of the above facts do not seem to be readily available in the literature. Let $V \in L^{\infty}\left(\mathbb{R}^{d}\right)$ and consider $H=\Psi(-\Delta)+V$. This operator is bounded from below, and self-adjoint on the dense domain $\operatorname{Dom}(\Psi(-\Delta)) \subset L^{2}\left(\mathbb{R}^{d}\right)$, with core $C_{\mathrm{c}}^{\infty}\left(\mathbb{R}^{d}\right)$. For a bounded open set $\mathcal{D} \subset \mathbb{R}^{d}$ we define the non-local Schrödinger operator $H^{\mathcal{D}, V}$ as the Friedrichs extension of $\left.H\right|_{C_{\mathrm{c}}^{\infty}(\mathcal{D})}$. Also, define

$$
T_{t}^{\mathcal{D}, V} f(x)=\mathbb{E}^{x}\left[e^{-\int_{0}^{t} V\left(X_{s}\right) d s} f\left(X_{t}\right) \mathbb{1}_{\left\{\tau_{D}>t\right\}}\right], \quad x \in \mathcal{D}, t>0, f \in L^{2}(\mathcal{D}) .
$$

We denote $L^{p}$ norm on $\mathcal{D}$ by $\|\cdot\|_{p, \mathcal{D}}$, whereas $\|\cdot\|_{p}$ denotes the $L^{p}$ norm on $\mathbb{R}^{d}$. We show the following properties.

Lemma 3.1. Consider the operators $H^{\mathcal{D}, V}$ and $T_{t}^{\mathcal{D}, V}, t>0$, and let $\left(S_{t}^{\Psi}\right)_{t \geq 0}$ be the subordinator corresponding to the Bernstein function $\Psi \in \mathcal{B}_{0}$. Suppose that $\Psi$ satisfies the Hartman-Wintner condition

$$
\lim _{|u| \rightarrow \infty} \frac{\Psi\left(|u|^{2}\right)}{\log |u|}=\infty
$$

The following hold:

(i) Every $T_{t}^{\mathcal{D}, V}$ is an integral operator and we have the representation

$$
\begin{aligned}
T_{t}^{\mathcal{D}, V} f(x) & =\int_{\mathcal{D}} \mathbb{E}_{\mathbb{P}_{S}}^{0}\left[p_{S_{t}^{\Psi}}(x-y) \mathbb{E}_{0, S_{t}^{\Psi}}^{x, y}\left[e^{-\int_{0}^{t} V\left(B_{S_{s}^{\Psi}}\right) d s}\right] \mathbb{1}_{\left\{\tau_{D}>t\right\}}\right] f(y) d y \\
& =\int_{\mathcal{D}} T^{\mathcal{D}, V}(t, x, y) f(y) d y, \quad x \in \mathcal{D}, t \geq 0, f \in L^{2}(\mathcal{D})
\end{aligned}
$$

where $p_{t}(x)=(4 \pi t)^{-d / 2} e^{-\frac{|x|^{2}}{4 t}}$, and $\mathbb{E}_{0, S_{t}^{\Psi}}^{x, y}$ denotes expectation with respect to the Brownian bridge measure from $x$ at time 0 to $y$ at time $s$, evaluated at random time $s=S_{t}^{\Psi}$. Furthermore, for every $t>0$, we have $T^{\mathcal{D}, V}(t, x, y)=T^{\mathcal{D}, V}(t, y, x)$ for all $x, y \in \mathbb{R}^{d}$.

(ii) $\left\{T_{t}^{\mathcal{D}, V}: t \geq 0\right\}$ is a strongly continuous semigroup on $L^{p}(\mathcal{D}), p \geq 1$, with infinitesimal generator $-H^{\mathcal{D}, V}$.

(iii) Every $T_{t}^{\mathcal{D}, V}$ is a Hilbert-Schmidt operator on $L^{2}(\mathcal{D})$, for all $t>0$.

(iv) The map $(0, \infty) \times \mathcal{D} \times \mathcal{D} \ni(t, x, y) \mapsto T^{\mathcal{D}, V}(t, x, y) \in \mathbb{R}$ is continuous.

(v) If $\mathcal{D}$ is a bounded domain with outer cone property, then for every $f \in L^{\infty}(\mathcal{D})$ we have that $T_{t}^{\mathcal{D}, V} f$ continuous in $\overline{\mathcal{D}}$ with value 0 on the boundary, for every $t>0$.

Proof. (i) (3.5) follows from a standard conditioning argument, see [35, Lem. 3.4]. We define

$$
T^{\mathcal{D}, V}(t, x, y)=\mathbb{E}_{\mathbb{P}_{S}}^{0}\left[p_{S_{t}^{\Psi}}(x-y) \mathbb{E}_{0, S_{t}^{\Psi}}^{x, y}\left[e^{-\int_{0}^{t} V\left(B_{S_{s}^{\Psi}}\right) d s} \mathbb{1}_{\left\{\tau_{\mathcal{D}}>t\right\}}\right]\right]
$$

and show that

$$
T^{\mathcal{D}, V}(t, x, y)=T^{\mathcal{D}, V}(t, y, x), \quad \text { for } t>0 .
$$

Consider the Brownian bridge on the interval $\left[0, S_{t}^{\Psi}\right]$ starting with $x$ and ending at $y$ given by

$$
Z_{s}^{x, y}=\left(1-\frac{s}{S_{t}^{\Psi}}\right) x+\frac{s}{S_{t}^{\Psi}} y+B_{s}-\frac{s}{S_{t}^{\Psi}} B_{S_{t}^{\Psi}},
$$


where $\left(B_{t}\right)_{t \geq 0}$ is the Brownian motion running twice as fast as the standard Brownian motion, independent of the subordinator $\left(S_{t}^{\Psi}\right)_{t \geq 0}$. A change of variable gives

$$
\int_{0}^{t} V\left(Z_{S_{s}^{\Psi}}^{x, y}\right) \mathrm{d} s=\int_{0}^{t} V\left(Z_{S_{t-s}^{\Psi}}^{x, y}\right) \mathrm{d} s
$$

and we also have

$$
Z_{S_{s}^{\Psi}}^{x, y} \in \mathcal{D}, \forall s \in[0, t] \Longleftrightarrow Z_{S_{t-s}^{\Psi}}^{x, y} \in \mathcal{D}, \forall s \in[0, t] .
$$

Therefore to show (3.6) we only need to show that

$$
\left(\left.Z_{S_{t-.}^{\Psi}}^{x, y}\right|_{[0, t]}, S_{t}^{\Psi}\right) \stackrel{\mathrm{d}}{=}\left(\left.Z_{S^{\Psi}}^{y, x}\right|_{[0, t]}, S_{t}^{\Psi}\right)
$$

This can be shown by using the fact that for any Lévy process $\left(L_{t}\right)_{t \geq 0}$ starting at zero we have

$$
\left(L_{t-.}, L_{t}\right) \stackrel{\mathrm{d}}{=}\left(L_{t}-L ., L_{t}\right) .
$$

First we show (3.7) using (3.8). Since the Brownian motion is independent of $\left(S_{t}^{\Psi}\right)_{t \geq 0}$, we get the following equalities in distribution

$$
\begin{aligned}
Z_{S_{t-}^{\Psi}}^{x, y} & \stackrel{\mathrm{d}}{=}\left(1-\frac{S_{t}^{\Psi}-S_{\cdot}^{\Psi}}{S_{t}^{\Psi}}\right) x+\frac{S_{t}^{\Psi}-S_{\cdot}^{\Psi}}{S_{t}^{\Psi}} y+B_{S_{t}^{\Psi}-S_{\cdot}^{\Psi}}-\frac{S_{t}^{\Psi}-S_{\cdot}^{\Psi}}{S_{t}^{\Psi}} B_{S_{t}^{\Psi}} \\
& \stackrel{\mathrm{d}}{=} \frac{S_{\cdot}^{\Psi}}{S_{t}^{\Psi}} x+\left(1-\frac{S_{\cdot}^{\Psi}}{S_{t}^{\Psi}}\right) y-B_{S_{\cdot}^{\Psi}}+\frac{S_{\cdot}^{\Psi}}{S_{t}^{\Psi}} B_{S_{t}^{\Psi}} \\
& \stackrel{\mathrm{d}}{=} \frac{S_{\cdot}^{\Psi}}{S_{t}^{\Psi}} x+\left(1-\frac{S_{\cdot}^{\Psi}}{S_{t}^{\Psi}}\right) y+B_{S^{\Psi}}-\frac{S_{\cdot}^{\Psi}}{S_{t}^{\Psi}} B_{S_{t}^{\Psi}}=Z_{S^{\Psi}}^{y, x} .
\end{aligned}
$$

This proves (3.7). Next we come to (3.8). It suffices to show that the finite dimensional distributions coincide. Consider $t>s_{1}>s_{2}>\cdots>s_{k} \geq 0$ and $\xi_{i} \in \mathbb{R}$ for $i=1, \ldots, k+1$. Then it is seen that

$$
\begin{aligned}
& \xi_{1} L_{t-s_{1}}+\ldots+\xi_{k} L_{t-s_{k}}+\xi_{k+1} L_{t} \\
& \quad=\sum_{i \geq 1} \xi_{i} L_{t-s_{1}}+\sum_{i \geq 2}^{k+1} \xi_{i}\left(L_{t-s_{2}}-L_{t-s_{1}}\right)+\cdots+\left(\xi_{k}+\xi_{k+1}\right)\left(L_{t-s_{k}}-L_{t-s_{k-1}}\right)+\xi_{k+1}\left(L_{t}-L_{t-s_{k}}\right)
\end{aligned}
$$

and

$$
\begin{aligned}
& \xi_{1}\left(L_{t}-L_{s_{1}}\right)+\ldots+\xi_{k}\left(L_{t}-L_{s_{k}}\right)+\xi_{k+1} L_{t} \\
& \quad=\sum_{i \geq 1} \xi_{i}\left(L_{t}-L_{s_{1}}\right)+\sum_{i \geq 2}^{k+1} \xi_{i}\left(L_{s_{1}}-L_{s_{2}}\right)+\cdots+\left(\xi_{k}+\xi_{k+1}\right)\left(L_{s_{k+1}}-L_{s_{k}}\right)+\xi_{k+1} L_{s_{k}} .
\end{aligned}
$$

On the other hand,

$$
\left(L_{t-s_{1}}, L_{t-s_{2}}-L_{t-s_{1}}, \cdots, L_{t}-L_{t-s_{k}}\right) \stackrel{\mathrm{d}}{=}\left(L_{t}-L_{s_{1}}, L_{s_{1}}-L_{s_{2}}, \cdots, L_{s_{k}}\right) .
$$

Thus $\left(L_{t-s_{1}}, \cdots, L_{t-s_{k}}, L_{t}\right)$ has the same characteristic function as $\left(L_{t}-L_{s_{1}}, \cdots, L_{t}-L_{s_{k}}, L_{t}\right)$, implying (3.8).

(ii) We establish the Chapman-Kolmogorov relation

$$
T^{\mathcal{D}, V}(t+s, x, y)=\int_{\mathcal{D}} T^{\mathcal{D}, V}(t, x, u) T^{\mathcal{D}, V}(s, u, y) \mathrm{d} u, \quad t, s>0, x, y \in \mathbb{R}^{d} .
$$

Denote

$$
\Xi(r, z, y)=\mathbb{E}_{0, S_{r}^{\Psi}}^{z, y}\left[e^{-\int_{0}^{r} V\left(Z_{u}\right) \mathrm{d} u} \mathbb{1}_{\left\{\tau_{D}>r\right\}}\right]
$$


where $\left(Z_{t}\right)_{t \geq 0}$ denotes the Brownian bridge as defined above. Let $\left(\widetilde{S}_{t}^{\Psi}\right)_{t \geq 0}$ be a subordinator given by Bernstein function $\Psi$, independent of $S^{\Psi}, B, Z$. Then we have

$$
\begin{aligned}
& \mathbb{E}_{\mathbb{P}_{S}}^{0}\left[p_{S_{t+s}^{\Psi}}(x-y) \mathbb{E}_{0, S_{t+s}^{\Psi}}^{x, y}\left[e^{-\int_{0}^{t+s} V\left(Z_{S_{u}^{\Psi}}\right) \mathrm{d} u} \mathbb{1}_{\left\{\tau_{\mathcal{D}}>t+s\right\}}\right]\right] \\
& =\mathbb{E}_{\mathbb{P}_{S}}^{0}\left[p_{S_{t+s}^{\Psi}}(x-y) \mathbb{E}_{0, S_{t+s}^{\Psi}}^{x, y}\left[\mathbb{1}_{\left\{\tau_{\mathcal{D}}>t\right\}} e^{-\int_{0}^{t} V\left(Z_{S_{u}^{\Psi}}\right) \mathrm{d} u} \mathbb{E}_{0, S_{t+s}^{\Psi}-S_{t}^{\Psi}}\left[e^{-\int_{0}^{s} V\left(Z_{S_{u+t}^{\Psi}}, S_{t}^{\Psi}\right) \mathrm{d} u} \mathbb{1}_{\left\{\tau_{\mathcal{D}}>s\right\}}\right]\right]\right] \\
& =\mathbb{E}_{\mathbb{P}_{S}}^{0}\left[p_{S_{t}^{\Psi}+\widetilde{S}_{s}^{\Psi}}(x-y) \mathbb{E}_{0, S_{t}^{\Psi}+\widetilde{S}_{s}^{\Psi}}^{x, y}\left[\mathbb{1}_{\left\{\tau_{\mathcal{D}}>t\right\}} e^{-\int_{0}^{t} V\left(Z_{S_{u}^{\Psi}}\right) \mathrm{d} u} \mathbb{E}_{0, \widetilde{S}_{s}^{\Psi}}^{Z_{S^{\Psi}}, y}\left[e^{-\int_{0}^{s} V\left(Z_{\widetilde{S}_{u}^{\Psi}}\right) \mathrm{d} u_{1}} \mathbb{1}_{\left\{\tau_{\mathcal{D}}>s\right\}}\right]\right]\right] \\
& =\mathbb{E}_{\mathbb{P}_{S}}^{0}\left[p_{S_{t}^{\Psi}+\widetilde{S}_{s}^{\Psi}}(x-y) \mathbb{E}_{0, S_{t}^{\Psi}+\widetilde{S}_{s}^{\Psi}}^{x, y}\left[\mathbb{1}_{\left\{\tau_{\mathcal{D}}>t\right\}} e^{-\int_{0}^{t} V\left(Z_{S_{u}^{\Psi}}\right) \mathrm{d} u} \Xi\left(\widetilde{S}_{s}^{\Psi}, Z_{S_{t}^{\Psi}}, y\right)\right]\right] \\
& =\mathbb{E}_{\mathbb{P}_{S}}^{0}\left[p _ { S _ { t } ^ { \Psi } + \widetilde { S } _ { s } ^ { \Psi } } ( x - y ) \mathbb { E } _ { \mathbb { P } _ { W } } ^ { x } \left[\mathbb{1}_{\left\{\tau_{\mathcal{D}}>t\right\}} e^{-\int_{0}^{t} V\left(B_{S_{u}^{\Psi}}\right) \mathrm{d} u} \Xi\left(\widetilde{S}_{s}^{\Psi}, B_{S_{t}^{\Psi}}, y\right) p_{\widetilde{S}_{s}^{\Psi}}\left(B_{S_{t}^{\Psi}}-y\right) \frac{1}{\left.\left.p_{S_{t}^{\Psi}+\widetilde{S}_{s}^{\Psi}}(x-y)\right]\right]}\right.\right. \\
& =\mathbb{E}_{\mathbb{P}_{S}}^{0}\left[\mathbb{E}_{\mathbb{P}_{W}}^{x}\left[\mathbb{1}_{\left\{\tau_{\mathcal{D}}>t\right\}} e^{-\int_{0}^{t} V\left(B_{S_{u}^{\Psi}}\right) \mathrm{d} u} \Xi\left(\widetilde{S}_{s}^{\Psi}, B_{S_{t}^{\Psi}}, y\right) p_{\widetilde{S}_{s}^{\Psi}}\left(B_{S_{t}^{\Psi}}-y\right)\right]\right] \\
& =\mathbb{E}_{\mathbb{P}_{S}}^{0}\left[\mathbb{E}_{\mathbb{P}_{W}}^{x}\left[\mathbb{1}_{\left\{\tau_{\mathcal{D}}>t\right\}} e^{-\int_{0}^{t} V\left(B_{S_{u}^{\Psi}}\right) \mathrm{d} u} T^{\mathcal{D}, V}\left(s, X_{t}, y\right)\right]\right] \\
& =\int_{\mathcal{D}} T^{\mathcal{D}, V}(t, x, u) T^{\mathcal{D}, V}(s, u, y) \mathrm{d} u,
\end{aligned}
$$

where the first equality follows from the Markov property of Brownian bridge, in the fourth line we used [65, Prop. A.1], and the sixth line follows by taking expectation with respect to $\left(\widetilde{S}_{t}^{\Psi}\right)_{t \geq 0}$. Strong continuity follows along the line of [65, Prop. 3.3].

(iii) The symmetry of $T^{\mathcal{D}, V}(t, x, y)$ implies that $T_{t}^{\mathcal{D}, V}$ is a self-adjoint operator on $L^{2}(\mathcal{D})$. Let $q_{t}(x, y)$ be the transition density of $\left(X_{t}\right)_{t>0}$. Then the Hartman-Wintner condition (3.4) implies that for every $t>0, q_{t}(\cdot)$ is bounded and continuous [33,47] and therefore, $q_{t}(x, \cdot) \in L^{2}\left(\mathbb{R}^{d}\right)$. Indeed, for $t>0$,

$$
q_{2 t}(x, x)=\int_{\mathbb{R}^{d}} q_{t}(x-y) q_{t}(y-x) \mathrm{d} y=\int_{\mathbb{R}^{d}} q_{t}^{2}(x-y) \mathrm{d} y<\infty .
$$

The transition density for the process $\left(X_{t}\right)_{t \geq 0}$ killed upon the first exit from $\mathcal{D}$ is given by Hunt's formula

$$
q_{t}^{\mathcal{D}}(x, y)=q_{t}(x, y)-\mathbb{E}^{x}\left[q_{t-\tau_{\mathcal{D}}}\left(X_{\tau_{\mathcal{D}}}, y\right) \mathbb{1}_{\left\{t>\tau_{\mathcal{D}}\right\}}\right] \quad t>0, x, y \in \mathbb{R}^{d} .
$$

In particular, $q_{t}^{\mathcal{D}}(x, y) \leq q_{t}(x, y)$. Since $V$ is bounded, we obtain

$$
\left|T_{t}^{\mathcal{D}, V} f(x)\right| \leq e^{t\|V\|_{\infty}} \int_{\mathcal{D}}|f(y)| q_{t}^{\mathcal{D}}(x, y) \mathrm{d} y \leq e^{t\|V\|_{\infty}}\|f\|_{2, \mathcal{D}}\left\|q_{t}(x, \cdot)\right\|_{2} .
$$

Note that $\left\|q_{t}(x, \cdot)\right\|_{2}$ does not depend on $x$. Therefore

$$
\int_{\mathcal{D} \times \mathcal{D}}\left(T^{\mathcal{D}, V}(t, x, y)\right)^{2} \mathrm{~d} y \mathrm{~d} x \leq C_{t}
$$

with a constant $C_{t}>0$, implying that $T_{t}^{\mathcal{D}, V}$ is a Hilbert-Schmidt operator.

(iv) We claim that for every $t>0$ and $y \in \mathcal{D}$,

$$
x \mapsto T^{\mathcal{D}, V}(t, x, y) \quad \text { is continuous in } \mathcal{D} \text {. }
$$

To show (3.10), write

$$
T_{\varepsilon}^{\mathcal{D}, V}(t, x, y)=\mathbb{E}_{\mathbb{P}_{S}}^{0}\left[\int_{\mathbb{R}^{d}} p_{S_{\varepsilon}^{\Psi}}(x-z) p_{\widetilde{S}_{t-\varepsilon}^{\Psi}}(z-y) \Xi(t-\varepsilon, z, y)\right]
$$




$$
\begin{aligned}
& =\mathbb{E}_{\mathbb{P}_{S}}^{0}\left[\int_{\mathcal{D}} p_{S_{\varepsilon}^{\Psi}}(x-z) p_{\widetilde{S}_{t-\varepsilon}^{\Psi}}(z-y) \Xi(t-\varepsilon, z, y)\right] \\
& =\mathbb{E}_{\mathbb{P}_{S}}^{0}\left[\int_{\mathbb{R}^{d}} p_{S_{\varepsilon}^{\Psi}+\widetilde{S}_{t-\varepsilon}^{\Psi}}(x-y) \mathbb{E}^{x, y}\left[e^{-\int_{\varepsilon}^{t} V\left(Z_{u}\right) \mathrm{d} u_{1}} \mathbb{1}_{\left\{\tau_{\mathcal{D}} \circ \sigma_{\varepsilon}>t-\varepsilon\right\}}\right]\right] \\
& =\mathbb{E}_{\mathbb{P}_{S}}^{0}\left[\int_{\mathbb{R}^{d}} p_{S_{t}^{\Psi}}(x-y) \mathbb{E}^{x, y}\left[e^{-\int_{\varepsilon}^{t} V\left(Z_{u}\right) \mathrm{d} u_{1}} \mathbb{1}_{\left\{\tau_{\mathcal{D}} \circ \sigma_{\varepsilon}>t-\varepsilon\right\}}\right]\right]
\end{aligned}
$$

where $\sigma_{\varepsilon}$ denotes the $\varepsilon$-shift operator, and the third line above follows from [65, Cor. A.2]. It is straightforward to see that $(3.10)$ holds for $T_{\varepsilon}^{\mathcal{D}, V}$. On the other hand, $T_{\varepsilon}^{\mathcal{D}, V}(t, \cdot, y)$ converges to $T^{\mathcal{D}, V}(t, \cdot, y)$ as $\varepsilon \rightarrow 0$, uniformly on the compact subsets of $\mathcal{D}$, see for example, [65, eq. (3.21)]. This proves (3.10). The proof of (iv) can be completed employing a similar argument as in [65, Prop. 3.5] combining (3.9), (3.10) and (ii).

Finally we prove $(\mathrm{v})$. Denote $\tilde{f}(t, x)=T_{t}^{\mathcal{D}, V} f(x)$. In view of (iv) it is enough to show that for $x_{n} \rightarrow z \in \partial \mathcal{D}$ we have

$$
\lim _{n \rightarrow \infty}\left|\tilde{f}\left(t, x_{n}\right)\right|=0 .
$$

Since $f$ and $V$ are bounded, we obtain from (3.3) that

$$
\left|\tilde{f}\left(t, x_{n}\right)\right| \leq e^{\|V\|_{\infty} t}\|f\|_{\infty} \mathbb{P}^{x_{n}}\left(\tau_{\mathcal{D}}>t\right) .
$$

Since $z \in \mathcal{D}$ is regular, see the proof of $[15$, Lem. 2.9], we have

$$
\lim _{x_{n} \rightarrow z} \mathbb{P}^{x_{n}}\left(\tau_{\mathcal{D}}>t\right)=0 .
$$

By combining the above two equalities (3.11) follows.

Remark 3.1. We note that Lemma 3.1 can be obtained also for $\Psi$-Kato class potentials, which may have local singularities (see below). Also, further (such as contractivity, positivity improving etc) properties of $\left\{T_{t}^{\mathcal{D}, V}: t \geq 0\right\}$ can be shown, which is left to the reader. The lemma can further be extended for other non-local Schrödinger operators, involving more general isotropic Lévy processes.

From Lemma 3.1 it then follows that the Dirichlet-Schrödinger eigenvalue equation (1.3) is solved by a countable set of eigenvalues $\lambda_{1}^{\mathcal{D}, V}<\lambda_{2}^{\mathcal{D}, V} \leq \lambda_{3}^{\mathcal{D}, V} \leq \cdots \rightarrow \infty$ and a corresponding orthonormal set of $L^{2}(\mathcal{D})$-eigenfunctions, such that the principal eigenvalue is simple and the corresponding principal eigenfunction has a strictly positive version.

\subsection{The location of extrema}

In the remaining part of this article we shall assume that $\mathcal{D}$ is a bounded, convex set. In the following we will use a class of potentials, which are general enough to contain many interesting cases (such as Coulomb-type potentials), while being naturally suitable for defining Feynman-Kac semigroups. Consider the set of functions

$$
\mathcal{K}^{\Psi}=\left\{f: \mathbb{R} \rightarrow \mathbb{R}^{d}: f \text { is Borel measurable and } \lim _{t \downarrow 0} \sup _{x \in \mathbb{R}^{d}} \mathbb{E}^{x}\left[\int_{0}^{t}\left|f\left(X_{s}\right)\right| d s\right]=0\right\} .
$$

We say that the potential $V: \mathbb{R}^{d} \rightarrow \mathbb{R}$ belongs to $\Psi$-Kato class whenever it satisfies

$$
V_{-} \in \mathcal{K}^{\Psi} \quad \text { and } \quad V_{+} \in \mathcal{K}_{\text {loc }}^{\Psi}, \quad \text { with } \quad V_{+}=\max \{V, 0\}, V_{-}=\min \{V, 0\},
$$

where $V_{+} \in \mathcal{K}_{\text {loc }}^{\Psi}$ means that $V_{+} 1_{\mathcal{C}} \in \mathcal{K}^{\Psi}$ for all compact sets $\mathcal{C} \subset \mathbb{R}^{d}$, and $\left(X_{t}\right)_{t \geq 0}$ is the Lévy process generated by $\Psi(-\Delta)$. It is direct to see that $L_{\text {loc }}^{\infty}\left(\mathbb{R}^{d}\right) \subset \mathcal{K}_{\text {loc }}^{\Psi}$, moreover, by stochastic continuity of $\left(X_{t}\right)_{t \geq 0}$ also $\mathcal{K}_{\text {loc }}^{\Psi} \subset L_{\text {loc }}^{1}\left(\mathbb{R}^{d}\right)$. By standard arguments based on Khasminskii's Lemma, for a 
$\Psi$-Kato class potential $V$ it follows that there exist suitable constants $C_{1}(\Psi, V), C_{2}(\Psi, V)>0$ such that

$$
\sup _{x \in \mathbb{R}^{d}} \mathbb{E}^{x}\left[e^{-\int_{0}^{t} V\left(X_{s}\right) \mathrm{d} s}\right] \leq \sup _{x \in \mathbb{R}^{d}} \mathbb{E}^{x}\left[e^{\int_{0}^{t} V_{-}\left(X_{s}\right) \mathrm{d} s}\right] \leq C_{1} e^{C_{2} t}, \quad t>0 .
$$

For further details we refer to [36, Sect. 4] and [55].

For Bernstein functions we will use the following property repeatedly below, which has been introduced in [14].

Assumption 3.1. The function is said to satisfy a weak local scaling (WLSC) property with parameters $\mu>0$ and $\underline{c} \in(0,1]$, if

$$
\Psi(\gamma u) \geq \underline{c} \gamma^{\mu} \Psi(u), \quad u>0, \gamma \geq 1 .
$$

We will show some typical examples of Bernstein functions satisfying Assumption 3.1 further below in this section.

Now we present two expressions of the main result of this section. The first uses $\Psi$-Kato class potentials $V$ and a restricted class of $\Psi$, the second uses a more general class of Bernstein functions $\Psi$ and bounded potentials.

Theorem 3.1. Let $\Psi \in \mathcal{B}_{0}$ satisfy Assumption 3.1 with $\mu>0$ and $\underline{c} \in(0,1]$. Let $V \in \mathcal{K}^{\Psi}$ be a $\Psi$-Kato class potential, with $V^{-} \in L^{p}\left(\mathbb{R}^{d}\right), p>\frac{d}{2 \mu}$. Also, let $\varphi$ be a non-zero solution of (1.3) at eigenvalue $\lambda^{V, \mathcal{D}}$. Assume that $|\varphi|$ attains a global maximum at $x^{*} \in \mathcal{D}$, and denote $r=\operatorname{dist}\left(x^{*}, \partial \mathcal{D}\right)$ and $\eta=1-\frac{d}{2 \mu p}$. Then there exists a constant $\Theta_{1}>0$, dependent on $d, \mu, \underline{c}, \eta$, inrad $\mathcal{D}$, and $a$ constant $\Theta_{2}>0$, dependent on $\eta$ only, such that

$$
\Theta_{1}\left\|V^{-}\right\|_{p}^{1 / \eta}-\inf _{\mathcal{D}} V^{+}+\lambda^{V, \mathcal{D}} \geq \Theta_{2} \Psi\left(r^{-2}\right) .
$$

The proof of Theorem 3.1 is simpler if the potential $V$ is bounded. Moreover, one can allow a larger class of $\Psi$, not necessarily satisfying WLSC, and the dependence of $\Theta_{1}$ on the domain parameters can be waived when $V \in L^{\infty}(\mathcal{D})$. This is obtained in the following theorem.

Theorem 3.2. Let $\Psi \in \mathcal{B}_{0}, V \in L^{\infty}\left(\mathbb{R}^{d}\right)$, and $\varphi$ be a non-zero solution of (1.3) at eigenvalue $\lambda^{V, \mathcal{D}}$. Assume that $|\varphi|$ attains a global maximum at $x^{*} \in \mathcal{D}$, and denote $r=\operatorname{dist}\left(x^{*}, \partial \mathcal{D}\right)$. Then there exists a universal constant $\theta>0$, independent of $\mathcal{D}, x^{*}, V, \Psi$ and the dimension $d$, such that

$$
\left\|V^{-}\right\|_{\infty, \mathcal{D}}-\inf _{\mathcal{D}} V^{+}+\lambda^{V, \mathcal{D}} \geq \theta \Psi\left(r^{-2}\right)
$$

with

$$
\theta=-\min _{\kappa>1} \frac{1}{\kappa} \log \left(1-F(-1)\left(1-e^{1-\kappa}\right)\right) \approx 0.0833
$$

where $F$ is the probability distribution function of a Gaussian random variable $N(0,2)$. In particular, if $\Psi$ is strictly increasing, then

$$
\operatorname{dist}\left(x^{*}, \partial \mathcal{D}\right) \geq \frac{1}{\sqrt{\Psi^{-1}\left(\frac{\left\|V^{-}\right\|_{\infty, \mathcal{D}}-\inf _{\mathcal{D}} V^{+}+\lambda^{V, \mathcal{D}}}{\theta}\right)}} .
$$

Next we turn to proving these theorems. For technical reasons we start by showing first the latter theorem.

Proof of Theorem 3.2. Let $\tau_{\mathcal{D}}$ be the first exit time of $\left(X_{t}\right)_{t \geq 0}$ from $\mathcal{D}$, as defined in (3.2). Using the eigenvalue equation and the representation (3.3), we have that

$$
\left|\varphi\left(x^{*}\right)\right| \leq e^{\lambda^{V, \mathcal{D}}} t_{\mathbb{E}^{x^{*}}}\left[e^{-\int_{0}^{t} V\left(X_{s}\right) \mathrm{d} s}\left|\varphi\left(X_{t}\right)\right| \mathbb{1}_{\left\{t<\tau_{\mathcal{D}}\right\}}\right] \leq\left|\varphi\left(x^{*}\right)\right| e^{\lambda^{V, \mathcal{D}}} t e^{\left(\left\|V^{-}\right\|_{\infty, \mathcal{D}}-\inf _{\mathcal{D}} V^{+}\right) t} \mathbb{P}^{x^{*}}\left(\tau_{\mathcal{D}}>t\right),
$$

that is,

$$
e^{t\left(\left\|V^{-}\right\|_{\infty, \mathcal{D}}-\inf _{\mathcal{D}} V^{+}+\lambda^{V, \mathcal{D}}\right)} \mathbb{P}^{x^{*}}\left(\tau_{\mathcal{D}}>t\right) \geq 1, \quad t \geq 0
$$


We choose

$$
t=\frac{\kappa}{\Psi\left(r^{-2}\right)}
$$

with a suitable $\kappa$, which will be justified below, and show that for this $t$ we have

$$
\mathbb{P}^{x^{*}}\left(\tau_{\mathcal{D}}>t\right)<\delta<1,
$$

where $\delta$ does not depend on $x^{*}, \mathcal{D}$.

Let $z \in \partial \mathcal{D}$ be such that $\operatorname{dist}\left(x^{*}, z\right)=r$, and consider the half-space $\mathcal{H} \subset \mathcal{D}^{c}$ intersecting $\mathcal{D}$ at $z$. Note that this is made possible by the convexity of $\mathcal{D}$, and

$$
\mathbb{P}^{x^{*}}\left(\tau_{\mathcal{D}} \leq t\right) \geq \mathbb{P}^{x^{*}}\left(X_{t} \in \mathcal{H}\right)
$$

holds. We assume with no loss of generality that $\mathcal{H}$ is perpendicular to the $x$-axis, $x^{*}=0$ and $z=(r, 0, \ldots, 0)$. This is possible, since we can inscribe a ball of radius $r$ in $\overline{\mathcal{D}}$ centered at $x^{*}$ and $\mathcal{H}$ would be a tangent plane to it at the point $z$. Therefore, we have for $s \geq r^{2}$ that

$$
\mathbb{P}_{W}^{x^{*}}\left(B_{s} \in \mathcal{H}\right)=\mathbb{P}_{W}^{0}\left(B_{s}^{1} \geq r\right)=\frac{1}{\sqrt{4 \pi}} \int_{\frac{r}{\sqrt{s}}}^{\infty} e^{-\frac{y^{2}}{4}} \mathrm{~d} y \geq \frac{1}{\sqrt{4 \pi}} \int_{1}^{\infty} e^{-\frac{y^{2}}{4}} \mathrm{~d} y=F(-1),
$$

where $\left(B_{t}^{1}\right)_{t \geq 0}$ denotes a one-dimensional Brownian motion running twice as fast as standard Brownian motion, and $F$ is the probability distribution function of a Gaussian random variable with mean 0 and variance 2 . Using the subordination formula (2.4) and the uniform estimate (3.21), we have

$$
\begin{aligned}
\mathbb{P}^{x^{*}}\left(X_{t} \in \mathcal{H}\right) & =\int_{0}^{\infty} \mathbb{P}_{W}^{x^{*}}\left(B_{s} \in \mathcal{H}\right) \mathbb{P}_{S}\left(S_{t}^{\Psi} \in \mathrm{d} s\right) \\
& \geq \int_{r^{2}}^{\infty} \mathbb{P}_{W}^{x^{*}}\left(B_{s} \in \mathcal{H}\right) \mathbb{P}_{S}\left(S_{t}^{\Psi} \in \mathrm{d} s\right) \geq F(-1) \mathbb{P}_{S}\left(S_{t}^{\Psi} \geq r^{2}\right)
\end{aligned}
$$

By (2.3) and (3.19) we have

$$
\mathbb{P}_{S}\left(S_{t}^{\Psi} \leq r^{2}\right)=\mathbb{P}_{S}\left(e^{-r^{-2} S_{t}^{\Psi}} \geq e^{-1}\right) \leq e \mathbb{E}_{\mathbb{P}_{S}}\left[e^{-r^{-2} S_{t}^{\Psi}}\right]=e^{1-t \Psi\left(r^{-2}\right)}=e^{1-\kappa} .
$$

Hence with $\kappa>1$ we obtain $\mathbb{P}_{S}\left(S_{t}^{\Psi} \leq r^{2}\right)<1$, and thus $(3.20)$ holds with $\delta=1-F(-1)\left(1-e^{1-\kappa}\right)$, independently on $r$. This then implies (3.15) with constant prefactor

$$
\theta_{\kappa}=-\frac{1}{\kappa} \log \left(1-F(-1)\left(1-e^{1-\kappa}\right)\right),
$$

which on optimizing over $\kappa$ gives the constant (3.16).

Proof of Theorem 3.1. The key estimate for the proof is the following improvement of (3.13): for any $\kappa_{1}>0$ there exists a constant $C_{1}>0$, dependent on $\kappa_{1}, d, \mu, \underline{c}$, satisfying for $t \in\left[0, \kappa_{1}\right]$ and $\vartheta>0$

$$
\sup _{x \in \mathbb{R}^{d}} \mathbb{E}^{x}\left[e^{\int_{0}^{t} \vartheta V^{-}\left(X_{s}\right) \mathrm{d} s}\right] \leq m_{\eta} e^{\left(C_{1} \vartheta\left\|V^{-}\right\|_{p} \Gamma(\eta)\right)^{1 / \eta} t},
$$

where $\eta=1-\frac{d}{2 \mu p}$ and $m_{\eta}$ depends only on $\eta$. First we complete the proof of the theorem assuming (3.22).

Choose $\kappa_{1}=\frac{2}{\Psi\left([\operatorname{inrad} \mathcal{D}]^{-2}\right)}$. Suppose that $r=\operatorname{dist}\left(x^{*}, \mathcal{D}\right)$ and let $t=\frac{2}{\Psi\left(r^{-2}\right)} \leq \kappa_{1}$. Then using (3.3) and Hölder inequality, we obtain for $\vartheta \geq 1$ that

$$
1 \leq e^{t\left(\lambda^{V, \mathcal{D}}-\inf _{\mathcal{D}} V^{+}\right)} \mathbb{E}^{x^{*}}\left[e^{\int_{0}^{t} \vartheta V^{-}\left(X_{s}\right) \mathrm{d} s}\right]^{1 / \vartheta}\left(\mathbb{P}^{x^{*}}\left(\tau_{\mathcal{D}}>t\right)\right)^{\frac{\vartheta-1}{\vartheta}} .
$$

Hence from (3.20), (3.22) and (3.23) we see that

$$
1 \leq \delta^{\frac{\vartheta-1}{\vartheta}}\left(m_{\eta}\right)^{1 / \vartheta} \exp \left(t\left[\lambda^{V, \mathcal{D}}-\inf _{\mathcal{D}} V^{+}+\frac{1}{\vartheta}\left(C_{1} \vartheta\left\|V^{-}\right\|_{p} \Gamma(\eta)\right)^{1 / \eta}\right]\right)
$$




$$
=\delta\left(\frac{m_{\eta}}{\delta}\right)^{1 / \vartheta} \exp \left(t\left[\lambda^{V, \mathcal{D}}-\inf _{\mathcal{D}} V^{+}+\frac{1}{\vartheta}\left(C_{1} \vartheta\left\|V^{-}\right\|_{p} \Gamma(\eta)\right)^{1 / \eta}\right]\right) .
$$

Since $\delta<1$ and $\lim _{\vartheta \rightarrow \infty}\left(\frac{m_{\eta}}{\delta}\right)^{1 / \vartheta}=1$, we can choose $\vartheta$ large enough such that

$$
\delta_{1}=\delta\left(\frac{m_{\eta}}{\delta}\right)^{1 / \vartheta}<1 .
$$

Thus we obtain

$$
\log \frac{1}{\delta_{1}} \leq t\left(\lambda^{V, \mathcal{D}}-\inf _{\mathcal{D}} V^{+}+\frac{1}{\vartheta}\left(C_{1} \vartheta\left\|V^{-}\right\|_{p} \Gamma(\eta)\right)^{1 / \eta}\right)
$$

implying

$$
\left(\frac{1}{2} \log \frac{1}{\delta_{1}}\right) \Psi\left(r^{-2}\right) \leq \lambda^{V, \mathcal{D}}-\inf _{\mathcal{D}} V^{+}+\frac{1}{\vartheta}\left(C_{1} \vartheta\left\|V^{-}\right\|_{p} \Gamma(\eta)\right)^{1 / \eta}
$$

This gives (3.14) for

$$
\Theta_{1}=\frac{1}{\vartheta}\left(C_{1} \vartheta \Gamma(\eta)\right)^{1 / \eta} \quad \text { and } \quad \Theta_{2}=\frac{1}{2} \log \frac{1}{\delta_{1}} .
$$

Now we proceed to establish (3.22). Since $\Psi$ has the WLSC property, the characteristic exponent $\Phi(r)=\Psi\left(r^{2}\right)$ also has the WLSC property, namely

$$
\Phi(\gamma u) \geq \underline{c} \gamma^{2 \mu} \Phi(u), \quad \text { for all } u>0 \text { and } \gamma \geq 1 .
$$

Thus by [14, Prop. 19] there exists a constant $K_{1}$, dependent on $d, \mu, \underline{c}$, satisfying

$$
q_{t}(x, y)=q_{t}(|x-y|) \leq K_{1}\left(\Phi^{-1}\left(\frac{1}{t}\right)\right)^{d}, \quad \forall t>0 .
$$

Here $q_{t}(x, y)$ denotes the transition density function of $\left(X_{t}\right)_{t \geq 0}$. On the other hand, from the WLSC property of $\Phi$ it follows that

$$
\Phi^{-1}(\lambda) \leq \lambda^{\frac{1}{2 \mu}} \frac{u}{\Phi(u)^{\frac{1}{2 \mu}}} \quad \text { for all } \lambda \geq \Phi(u), u>0 .
$$

Choose $\nu>0$ and denote $\nu_{1}=\frac{\nu}{(\Phi(\nu))^{\frac{1}{2 \mu}}}$. Then for $s \geq \Phi(\nu)$ we obtain

$$
\Phi^{-1}(s) \leq \nu_{1} s^{-2 \mu}
$$

Hence, using the above estimate in (3.24) we get that

$$
q_{t}(x, y) \leq K_{2} t^{-\frac{d}{2 \mu}}, \quad t \leq \frac{1}{\Psi\left(\nu^{2}\right)},
$$

where $K_{2}$ depends on $d, \mu$ and $\nu_{1}$. Let $\kappa_{1}$ be positive and choose $\nu=\sqrt{\Psi^{-1}\left(\frac{1}{\kappa_{1}}\right)}$. With this choice of $\nu$ we have from (3.26) that

$$
q_{t}(x, y) \leq K_{2} t^{-\frac{d}{2 \mu}}, \quad t \leq \kappa_{1} .
$$

For every $t \in\left(0, \kappa_{1}\right]$ and $f \in L^{p}\left(\mathbb{R}^{d}\right)$ we have

$$
\begin{aligned}
\mathbb{E}^{x}\left[f\left(X_{t}\right)\right] & \leq\|f\|_{p}\left[\int_{\mathbb{R}^{d}}\left(q_{t}(|x-y|)\right)^{p^{\prime}} \mathrm{d} y\right]^{1 / p^{\prime}} \\
& \leq K_{2}^{1 / p}\|f\|_{p} t^{-\frac{d}{2 \mu p}}\left[\int_{\mathbb{R}^{d}} q_{t}(|x-y|) \mathrm{d} y\right]^{1 / p^{\prime}}=K_{3}\|f\|_{p} t^{-\frac{d}{2 \mu p}}
\end{aligned}
$$

where $p^{\prime}=\frac{p}{p-1}, K_{3}=K_{2}^{1 / p}$, and in the second line above we used (3.27). Let now $0 \leq s_{1} \leq \ldots \leq s_{k}$, $k \in \mathbb{N}$. Using the Markov property of $\left(X_{t}\right)_{t \geq 0}$ with respect to its natural filtration $\left(\mathcal{F}_{t}\right)_{t \geq 0}$, for $f \geq 0$ we obtain

$$
\mathbb{E}^{x}\left[f\left(X_{s_{1}}\right) \cdots f\left(X_{s_{k}}\right)\right]=\mathbb{E}^{x}\left[f\left(X_{s_{1}}\right) \cdots f\left(X_{s_{k-1}}\right) \mathbb{E}^{x}\left[f\left(X_{s_{k}}\right) \mid \mathcal{F}_{s_{k-1}}\right]\right]
$$




$$
\begin{aligned}
& =\mathbb{E}^{x}\left[f\left(X_{s_{1}}\right) \cdots f\left(X_{s_{k-1}}\right) \mathbb{E}^{X_{s_{k}}}\left[f\left(X_{s_{k}-s_{k-1}}\right)\right]\right. \\
& \leq K_{3}\|f\|_{p}\left(s_{k}-s_{k-1}\right)^{-\frac{d}{2 \mu p}} \mathbb{E}^{x}\left[f\left(X_{s_{1}}\right) \cdots f\left(X_{s_{k-1}}\right)\right] \\
& \leq \ldots \leq\left(K_{3}\|f\|_{p}\right)^{k} s_{1}^{-\frac{d}{2 \mu p}}\left(s_{2}-s_{1}\right)^{-\frac{d}{2 \mu p}} \cdots\left(s_{k}-s_{k-1}\right)^{-\frac{d}{2 \mu p}} .
\end{aligned}
$$

Hence (compare [55, Lem. 4.51] in the second edition)

$$
\begin{aligned}
\mathbb{E}^{x}\left[\frac{1}{k !}\left(\int_{0}^{t} f\left(X_{s}\right) \mathrm{d} s\right)^{k}\right] \\
\leq \int_{0}^{t} \mathrm{~d} s_{1} \int_{s_{1}}^{t} \mathrm{~d} s_{2} \ldots \int_{s_{k}}^{t} \mathrm{~d} s_{k} \mathbb{E}^{x}\left[f\left(X_{s_{1}}\right) f\left(X_{s_{2}}\right) \ldots f\left(X_{s_{k}}\right)\right] \\
\leq K_{3}^{k}\|f\|_{p}^{k} \int_{0}^{t} \mathrm{~d} s_{1} \int_{s_{1}}^{t} \mathrm{~d} s_{2} \ldots \int_{s_{k}}^{t} \mathrm{~d} s_{k} s_{1}^{-\frac{d}{2 \mu p}}\left(s_{2}-s_{1}\right)^{-\frac{d}{2 \mu p}} \cdots\left(s_{k}-s_{k-1}\right)^{-\frac{d}{2 \mu p}} \\
=\frac{\left(K_{3}\|f\|_{p} t^{\eta} \Gamma(\eta)\right)^{k}}{\Gamma(1+k \eta)}, \quad t \leq \kappa_{1},
\end{aligned}
$$

where $\eta=1-\frac{d}{2 \mu p}>0$, by our choice of $p$. Recall the Mittag-Leffler function

$$
\mathcal{M}_{\beta}(x)=\sum_{k=0}^{\infty} \frac{x^{k}}{\Gamma(1+\beta k)}
$$

(see [30] for definitions and properties). We find by the above that for $t \in\left[0, \kappa_{1}\right]$,

$$
\sup _{x \in \mathbb{R}^{d}} \mathbb{E}^{x}\left[e^{\int_{0}^{t} f\left(X_{s}\right) \mathrm{d} s}\right] \leq \mathcal{M}_{\eta}\left(K_{3}\|f\|_{p} t^{\eta} \Gamma(\eta)\right) .
$$

It is also known that for some constant $m_{\eta}$, dependent only on $\eta$,

$$
\mathcal{M}_{\eta}(x) \leq m_{\eta} e^{x^{1 / \eta}}, \quad x \geq 0,
$$

holds. Thus, using (3.28) we have for $t \leq \kappa_{1}$ that

$$
\sup _{x \in \mathbb{R}^{d}} \mathbb{E}^{x}\left[e^{\int_{0}^{t} f\left(X_{s}\right) \mathrm{d} s}\right] \leq m_{\eta} e^{\left(K_{3}\|f\|_{p} \Gamma(\eta)\right)^{\frac{1}{\eta}} t} .
$$

Putting $f=\vartheta V^{-}$in (3.29), we obtain (3.22).

The dependence of $\Theta_{1}$ on inrad $\mathcal{D}$ is due to the factor

$$
\nu_{1}=\frac{\nu}{(\Phi(\nu))^{\frac{1}{2 \mu}}},
$$

which appears in (3.25). For $\Psi(u)=u^{\alpha / 2}$, however, $\nu_{1}$ does not depend on $\nu$. Thus we have the following improvement to Theorem 3.1.

Corollary 3.1. Suppose that $\Psi(u)=u^{\alpha / 2}$. Moreover, assume that $V$ is a $\Psi$-Kato class function with $V^{-} \in L^{p}\left(\mathbb{R}^{d}\right), p>\frac{d}{\alpha}$. Let $\varphi$ be a non-zero solution of $(1.3)$ at eigenvalue $\lambda^{V, \mathcal{D}}$. Assume that $|\varphi|$ attains a global maximum at $x^{*} \in \mathcal{D}$, and denote $r=\operatorname{dist}\left(x^{*}, \partial \mathcal{D}\right)$. Then there exist $\Theta_{1}$, dependent on $d, \alpha, \underline{c}, \eta$, and $\Theta_{2}$, dependent on $\eta$, where $\eta=1-\frac{d}{\alpha p}$, such that

$$
\Theta_{1}\left\|V^{-}\right\|_{p}^{1 / \eta}-\inf _{\mathcal{D}} V^{+}+\lambda^{V, \mathcal{D}} \geq \Theta_{2} \Psi\left(r^{-2}\right)
$$

holds. 
Remark 3.2. For classical Schrödinger operators we have $\Psi(u)=u$, for which Theorem 3.2 implies (1.1), possibly with a different constant $c$. Also, for fractional Schrödinger operators we have $\Psi(u)=u^{\alpha / 2}$, which reproduces the result obtained in [8]. Formulae (3.15)-(3.17) equally apply for $V \equiv 0$, in which case the statement refers to the Dirichlet eigenfunctions and eigenvalues.

Example 3.1. Some important examples of $\Psi$ satisfying Assumption 3.1 include:

(i) $\Psi(u)=u^{\alpha / 2}, \alpha \in(0,2]$, with $\mu=\frac{\alpha}{2}$.

(ii) $\Psi(u)=\left(u+m^{2 / \alpha}\right)^{\alpha / 2}-m, m>0, \alpha \in(0,2)$, with $\mu=\frac{\alpha}{2}$.

(iii) $\Psi(u)=u^{\alpha / 2}+u^{\beta / 2}, \alpha, \beta \in(0,2]$, with $\mu=\frac{\alpha}{2} \wedge \frac{\beta}{2}$.

(iv) $\Psi(u)=u^{\alpha / 2}(\log (1+u))^{\beta / 2}, \alpha \in(0,2), \beta \in(0,2-\alpha)$, with $\mu=\frac{\alpha}{2}$.

(v) $\Psi(u)=u^{\alpha / 2}(\log (1+u))^{-\beta / 2}, \alpha \in(0,2], \beta \in[0, \alpha)$ with $\mu=\frac{\alpha-\beta}{2}$. (Since for $\gamma \geq 1, u>0$, $(1+u)^{\gamma} \geq(1+\gamma u)$ holds, we have $\gamma^{\beta / 2}(\log (1+u))^{\beta / 2} \geq(\log (1+\gamma u))^{\beta / 2}$.)

\subsection{Consequences on the spectrum}

The above theorems have a number of implications on the eigenvalues and related quantities. Here we discuss these implications involving an interplay of survival times of paths and geometric features.

Corollary 3.2. Let $\varphi$ be an eigenfunction corresponding to eigenvalue $\lambda^{V, \mathcal{D}}$ of $\Psi(-\Delta)+V$ under the conditions of Theorem 3.2. Suppose that $\lambda^{V, \mathcal{D}}>0$. Then we have

$$
\int_{0}^{\infty} \mathbb{E}^{x^{*}}\left[e^{-\int_{0}^{t} V\left(X_{s}\right) \mathrm{d} s} \mathbb{1}_{\left\{\tau_{\mathcal{D}}>t\right\}}\right] \mathrm{d} t \geq \frac{1}{\lambda^{V, \mathcal{D}}}
$$

where $x^{*}$ is a maximizer of $|\varphi|$ in $\mathcal{D}$.

Proof. From the proof of Theorem 3.2 we have

$$
\mathbb{E}^{x^{*}}\left[e^{-\int_{0}^{t} V\left(X_{s}\right) \mathrm{d} s} \mathbb{1}_{\left\{\tau_{\mathcal{D}}>t\right\}}\right] \geq e^{-\lambda^{V, \mathcal{D}} t}, \quad t \geq 0 .
$$

By integrating both sides in $t$ on $(0, \infty)$, we obtain (3.30).

Note that the left hand side of (3.30) gives the mean survival time of the process $\left(X_{t}\right)_{t \geq 0}$ starting from $x^{*}$, perturbed by the potential $V$, thus the above result gives a probabilistic bound on the Dirichlet-Schrödinger eigenvalues.

Remark 3.3. Using the trivial bound

$$
\operatorname{dist}\left(x^{*}, \partial \mathcal{D}\right) \leq \operatorname{inrad} \mathcal{D}
$$

involving the inradius of $\mathcal{D}$, we get the geometric constraint

$$
\lambda^{V, \mathcal{D}} \geq \theta \Psi\left(\frac{1}{(\operatorname{inrad} \mathcal{D})^{2}}\right)-\left\|V^{-}\right\|_{\infty}+\inf _{\mathcal{D}} V^{+}
$$

on the bottom of the spectrum.

Remark 3.4. Since $\Psi^{-1}$ is an increasing function, the bound (3.17) can be interpreted as saying that if the potential is not strong enough, the global extrema of $\varphi$ cannot be too close to the boundary. Intuitively it is clear that one can decrease $\operatorname{dist}\left(x^{*}, \partial \mathcal{D}\right)$, for instance, by a potential which has a hole close to the boundary, that is deep enough to make the process stay in that region with a sufficiently high probability, preventing it to hit the boundary too soon and get killed. It is seen that the condition only requires sufficient strength of the potential and no details on its local behaviour. There is also a probabilistic interpretation of relation (3.15). From [63, Rem. 4.8] we find that

$$
c_{1} \mathbb{E}^{x}\left[\tau_{\mathcal{B}_{r}(x)}\right] \leq \frac{1}{\Psi\left(r^{-2}\right)} \leq c_{2} \mathbb{E}^{x}\left[\tau_{\mathcal{B}_{r}(x)}\right]
$$


for some constants $c_{1}, c_{2}>0$ depending only on $d$. A combination with (3.30) then implies that the inequality makes a comparison of the mean survival time of the process starting from $x^{*}$ perturbed by the potential with the mean survival time of the free (unperturbed) process, involving the proportionality constant $\theta$. Note that since the principal eigenfunction $\varphi_{1}$ is strictly positive, by the Doob $h$-transform $f \mapsto \varphi_{1} f, f \in L^{2}(\mathcal{D})$, we can construct a random process generated by the operator $\widetilde{H} f=\frac{1}{\varphi_{1}} H\left(\varphi_{1} f\right)$ whose stationary measure is $\varphi_{1}^{2} d x$. The location $x^{*}$ of a global maximum of $\varphi_{1}$ then corresponds to a mode of the stationary density of the process conditioned never to exit the domain $\mathcal{D}$.

Next we consider the principal Dirichlet eigenvalues in the absence of a potential.

Corollary 3.3. Let $V=0$ and consider the principal eigenvalue $\lambda_{1}^{\mathcal{D}}$ of the Dirichlet problem (1.3) for $\Psi(-\Delta)$.

(i) We have

$$
\lambda_{1}^{\mathcal{D}} \geq\left(\frac{\Gamma(p+1)}{\sup _{x \in \mathcal{D}} \mathbb{E}^{x}\left[\tau_{\mathcal{D}}^{p}\right]}\right)^{1 / p}
$$

for every $p \geq 1$.

(ii) Let $\Psi \in \mathcal{B}_{0}$ be a complete Bernstein function. Then there exist positive universal constants $C_{1}, C_{2}$, dependent only on $d$, such that

$$
\frac{C_{1}}{\Psi\left([\operatorname{inrad} \mathcal{D}]^{-2}\right)} \leq \sup _{x \in \mathcal{D}} \mathbb{E}^{x}\left[\tau_{\mathcal{D}}\right] \leq \frac{C_{2}}{\Psi\left([\operatorname{inrad} \mathcal{D}]^{-2}\right)} .
$$

(iii) There exists a constant $C_{3}>0$, dependent on $d$, such that

$$
\frac{1}{\sup _{x \in \mathcal{D}} \mathbb{E}^{x}\left[\tau_{\mathcal{D}}\right]} \leq \lambda_{1}^{\mathcal{D}} \leq \frac{C_{3}}{\sup _{x \in \mathcal{D}} \mathbb{E}^{x}\left[\tau_{\mathcal{D}}\right]} .
$$

Proof. Let $p \geq 1$. To obtain (i) multiply both sides of (3.31) by $p t^{p-1}$ and integrate with respect to $t$ over $(0, \infty)$.

Next consider (ii). To prove (3.33) first note that by the domain monotonicity property we have

$$
\lambda_{1, \text { Lap }}^{\mathcal{D}} \leq \frac{\kappa_{1}}{[\operatorname{inrad} \mathcal{D}]^{2}},
$$

where $\kappa_{1}=\lambda_{1, \text { Lap }}^{\mathcal{B}}$ is the Dirichlet principal eigenvalue in the unit ball, and $\lambda_{1, \text { Lap }}^{\mathcal{D}}$ denotes the principal Dirichlet eigenvalue of the Laplacian in $\mathcal{D}$. Therefore by [23] we obtain

$$
\lambda_{1}^{\mathcal{D}} \leq \Psi\left(\lambda_{1, \text { Lap }}^{\mathcal{D}}\right) \leq \Psi\left(\frac{\kappa_{1}}{[\operatorname{inrad} \mathcal{D}]^{2}}\right)
$$

Thus, using (3.32) for $p=1$, we have

$$
\frac{1}{\Psi\left(\kappa_{1}^{-1}[\operatorname{inrad} \mathcal{D}]^{-2}\right)} \leq \sup _{x \in \mathcal{D}} \mathbb{E}^{x}\left[\tau_{\mathcal{D}}\right]
$$

From the Laplace transform of $\left(S_{t}^{\Psi}\right)_{t \geq 0}$ and the monotonicity of $\Psi$ it is seen that for every $\delta \geq 1$ we have

$$
\Psi(u) \leq \Psi(\delta u) \leq \delta \Psi(u), \quad \forall u \geq 0 .
$$

Thus by (3.35) we get the left hand side of (3.33) with $\kappa_{1}^{-1} \vee 1=C_{1}^{-1}$. To prove the converse implication we use a result from [59]. Note that since $\Psi$ is a complete Bernstein function, the process $\left(X_{t}\right)_{t \geq 0}$ has a transition density $q(t, x, y)=q(t, x-y)$. Moreover, $q(t, \cdot)$ is radially symmetric and decreasing. Denote by $r_{\mathcal{D}}=\operatorname{inrad} \mathcal{D}$ and define

$$
S_{\mathcal{D}}=\left\{x \in \mathbb{R}^{d}: x \in \mathbb{R}^{d-1} \times\left(-r_{\mathcal{D}}, r_{\mathcal{D}}\right)\right\} .
$$


Fix $t>0$ and $z_{0} \in \mathcal{D}$. By $\tau_{S_{\mathcal{D}}}$ we denote the first exit time from $S_{\mathcal{D}}$. Then

$$
\begin{aligned}
\mathbb{P}^{z_{0}}\left(\tau_{\mathcal{D}}>t\right) & =\lim _{m \rightarrow \infty} \mathbb{P}^{z_{0}}\left(X_{\frac{t}{m}} \in \mathcal{D}, X_{\frac{2 t}{m}} \in \mathcal{D}, \ldots, X_{\frac{m t}{m}} \in \mathcal{D}\right) \\
& =\lim _{m \rightarrow \infty} \int_{\mathcal{D}} \int_{\mathcal{D}} \cdots \int_{\mathcal{D}} \Pi_{j=1}^{m} q\left(\frac{t}{m}, z_{j}-z_{j-1}\right) \mathrm{d} z_{1} \mathrm{~d} z_{2} \cdots \mathrm{d} z_{m} \\
& \leq \lim _{m \rightarrow \infty} \int_{S_{\mathcal{D}}} \int_{S_{\mathcal{D}}} \cdots \int_{S_{\mathcal{D}}} q\left(\frac{t}{m}, 0, z_{1}\right) \Pi_{j=2}^{m} q\left(\frac{t}{m}, z_{j}-z_{j-1}\right) \mathrm{d} z_{1} \mathrm{~d} z_{2} \cdots \mathrm{d} z_{m} \\
& =\lim _{m \rightarrow \infty} \mathbb{P}^{0}\left(X_{\frac{t}{m}} \in S_{\mathcal{D}}, X_{\frac{2 t}{m}} \in S_{\mathcal{D}}, \ldots, X_{\frac{m t}{m}} \in S_{\mathcal{D}}\right)=\mathbb{P}^{0}\left(\tau_{S_{\mathcal{D}}}>t\right),
\end{aligned}
$$

where in the inequality above we used [59, Th. 1.2]. On the other hand, the first exit time $\tau_{S_{\mathcal{D}}}$ starting from 0 is equal in distribution to the first exit time of a one-dimensional subordinate Brownian motion from the interval $\mathcal{B}_{r_{\mathcal{D}}}=\left(-r_{\mathcal{D}}, r_{\mathcal{D}}\right)$ starting from 0 . Let $\left(B_{S_{t}^{\Psi}}^{1}\right)_{t \geq 0}$ be a onedimensional subordinate Brownian motion, and $\tau_{r_{\mathcal{D}}}$ be its first exit time from $\mathcal{B}_{r_{\mathcal{D}}}$. The above estimate gives

$$
\sup _{x \in \mathcal{D}} \mathbb{E}^{x}\left[\tau_{\mathcal{D}}\right] \leq \mathbb{E}^{0}\left[\tau_{r_{\mathcal{D}}}\right]
$$

Since the Lévy exponent of $\left(B_{S_{t}^{\Psi}}^{1}\right)_{t \geq 0}$ is given by $\Psi\left(u^{2}\right)$, we obtain from [63, Rem. 4.8] and (3.35) that

$$
\mathbb{E}^{0}\left[\tau_{r_{\mathcal{D}}}\right] \leq \frac{C_{2}}{\Psi\left(r_{\mathcal{D}}^{-2}\right)},
$$

for some universal constant $C_{2}$. Hence using (3.36) and the above estimate we obtain the right hand side of (3.33).

Finally, consider (iii). In view of (3.32) we only need to show the right hand side of (3.34). Using (3.36) and the estimate above, we get that

$$
\sup _{x \in \mathcal{D}} \mathbb{E}^{x}\left[\tau_{\mathcal{D}}\right] \leq \frac{C_{2}}{\Psi\left(r_{\mathcal{D}}^{-2}\right)}=\frac{C_{2} \lambda_{1}^{\mathcal{D}}}{\Psi\left(r_{\mathcal{D}}^{-2}\right)} \frac{1}{\lambda_{1}^{\mathcal{D}}} .
$$

On the other hand, using [23] and the domain monotonicity of the principal eigenvalue, we obtain

$$
\lambda_{1}^{\mathcal{D}} \leq \Psi\left(\lambda_{1, \mathrm{Lap}}^{\mathcal{D}}\right) \leq \Psi\left(\frac{\kappa_{1}}{r_{\mathcal{D}}^{2}}\right),
$$

where $\kappa_{1}=\lambda_{1, \text { Lap }}^{\mathcal{B}}$. A combination with (3.37) gives

$$
\sup _{x \in \mathcal{D}} \mathbb{E}^{x}\left[\tau_{\mathcal{D}}\right] \leq C_{2} \sup _{s \in(0, \infty)} \frac{\Psi\left(\kappa_{1} s\right)}{\Psi(s)} \frac{1}{\lambda_{1}^{\mathcal{D}}} .
$$

Hence using (3.38) and (3.35) we find

$$
\sup _{x \in \mathcal{D}} \mathbb{E}^{x}\left[\tau_{\mathcal{D}}\right] \leq C_{2}\left(1 \vee \kappa_{1}\right) \frac{1}{\lambda_{1}^{\mathcal{D}}}
$$

This completes the proof of (3.34).

Remark 3.5. The bound in (3.32) implies for $p=1$ the well-known relation

$$
\lambda_{1}^{\mathcal{D}} \geq \frac{1}{\sup _{x \in \mathcal{D}} \mathbb{E}^{x}\left[\tau_{\mathcal{D}}\right]},
$$

between the principal Dirichlet eigenvalue and the mean survival time in the domain, first obtained by Donsker and Varadhan for diffusion processes [26, eq. (1.2)]. For the classical case $\Psi(u)=u$, the lower bound

$$
\sup _{x \in \mathcal{D}} \mathbb{E}^{x}\left[\tau_{\mathcal{D}}\right] \lambda_{1}^{\mathcal{D}} \geq 1
$$


is known to be sharp for any any bounded domain $\mathcal{D}$ in $\mathbb{R}^{d}$ [34]. The moment estimates

$$
\sup _{x \in \mathcal{D}} \mathbb{E}^{x}\left[\tau_{\mathcal{D}}^{p}\right] \geq \frac{\Gamma(p+1)}{\left(\lambda_{1}^{\mathcal{D}}\right)^{p}}, \quad p \geq 1
$$

have also an independent interest, giving bounds on the (integer and fractional) moments of the mean exit time from $\mathcal{D}$ for subordinate Brownian motion, which were not known before. Also, using the same (3.31), it follows that $\tau_{\mathcal{D}}$ has $p$-exponential moments of order $p<\lambda_{1}^{\mathcal{D}}$, and we have the bound

$$
\sup _{x \in \mathcal{D}} \mathbb{E}^{x}\left[e^{p \tau_{\mathcal{D}}}\right] \geq \frac{p}{\lambda_{1}^{\mathcal{D}}-p}, \quad p<\lambda_{1}^{\mathcal{D}}
$$

Remark 3.6. There is much important work on estimates similar to (3.33)-(3.34) for cases when the reference domain is a simply connected set in $\mathbb{R}^{2}$ and the stochastic process is Brownian motion. Much effort has been made on finding the best possible universal constants for these estimates; see, for instance, $[1,3]$ and the references therein. For similar estimates for symmetric stable processes we refer to $[5,59]$. Corollary 3.3 extends the earlier results to subordinate Brownian motion, possibly with non-optimal constants.

Remark 3.7 (Hot-spots). In the literature the location where the solution of the heat equation in a bounded domain at a given time attains its maximum is referred to as a hot-spot. Identifying possible hot spots in a convex domain is known to be quite challenging and there is an extensive literature in this direction. In the case of Neumann boundary conditions the solution approaches the second eigenfunction on the long run, and the so called Rauch-conjecture states that this eigenfunction attains its maximum on the boundary of the domain, thus the hot-spots in this case are expected to be located on the edge. This conjecture turned out to be more involved and false in general, but it has been proven to hold under specific assumptions on the domain, see [2, 19, 39] and references therein. For Dirichlet boundary conditions the situation is different as now the solution of the heat equation tends to principal eigenfunction as time goes to infinity, and the hot-spot becomes its maximizer, away from the boundary. In [17, Th. 2.8] it is shown that there exists a constant $c$, dependent only on $d$, such that for any bounded convex set $\mathcal{D}$ one has

$$
\operatorname{dist}\left(x^{*}, \partial \mathcal{D}\right) \geq c \operatorname{inrad} \mathcal{D}\left(\frac{\operatorname{inrad} \mathcal{D}}{\operatorname{diam} \mathcal{D}}\right)^{d^{2}-1},
$$

where $x^{*}$ denotes a hot-spot of the Laplacian in $\mathcal{D}$ with Dirichlet boundary condition. Note that Theorem 3.2 improves this result substantially. We single this out in the following result.

Corollary 3.4 (Hot-spots). Let $\Psi(u)=u$, and $\lambda_{1}^{\mathcal{D}}$ be the principal Dirichlet eigenvalue of the Laplacian for the domain $\mathcal{D}$, and $\mathcal{B}$ denote the unit ball centered in the origin. Then

$$
\operatorname{dist}\left(x^{*}, \partial \mathcal{D}\right) \geq \sqrt{\frac{\theta}{\lambda_{1}^{\mathcal{B}}}} \operatorname{inrad} \mathcal{D},
$$

Proof. By the domain monotonicity property we have

$$
\lambda_{1}^{\mathcal{D}} \leq \frac{1}{(\operatorname{inrad} \mathcal{D})^{2}} \lambda_{1}^{\mathcal{B}}
$$

Using (3.17), the result follows, possibly with a non-optimal constant.

Remark 3.8 (Universal upper bound on the distance of maximizer). It is not difficult to see that a reverse inequality to (3.15) does not hold. Consider the domain $\mathcal{D}=[0, \pi]^{2}$ and the Laplace operator. Then $\varphi_{n}(x, y)=\sin ((2 n+1) x) \sin (y), n \in \mathbb{N}$, is an eigenfunction with eigenvalue $\lambda_{n}=$ 
$(2 n+1)^{2}+1$. Note that $\left|\varphi_{n}\left(\frac{\pi}{2}, \frac{\pi}{2}\right)\right|=1$ and $\operatorname{dist}\left(\left(\frac{\pi}{2}, \frac{\pi}{2}\right), \partial \mathcal{D}\right)=\frac{\pi}{2}$. Thus there is no $c>0$ such that

$$
\frac{\pi}{2}=\operatorname{dist}\left(\left(\frac{\pi}{2}, \frac{\pi}{2}\right), \partial \mathcal{D}\right) \leq\left(\frac{c}{\lambda_{n}}\right)^{1 / 2}, \quad \text { for all } n \in \mathbb{N} .
$$

We note that $z_{n}=\left(\frac{\pi}{(2 n+1) 2}, \frac{\pi}{2}\right)$ is also a maximizer of $\varphi_{n}$ and $\operatorname{dist}\left(z_{n}, \partial \mathcal{D}\right) \sim \lambda_{n}^{-1 / 2}$. Therefore an interesting open question is whether there exists a universal constant $c$ such that the $c \lambda_{n}^{-1 / 2}$ neighbourhood of $\partial \mathcal{D}$ contains an extremum of the Dirichlet eigenfunction $\varphi_{n}$.

Inequality (3.17) has another important consequence, which we single out next. Assume that $\Psi$ is strictly increasing in $(0, \infty)$, denote the Lebesgue measure of $\mathcal{D}$ by $|\mathcal{D}|$, and $|\mathcal{B}|=\omega_{d}$.

Corollary 3.5 (Faber-Krahn inequality). Under the conditions of Theorem 3.2 we have

$$
|\mathcal{D}|\left(\Psi^{-1}\left(\frac{\left\|V^{-}\right\|_{\infty}-\inf _{\mathcal{D}} V^{+}+\lambda^{V, \mathcal{D}}}{\theta}\right)\right)^{d / 2} \geq \omega_{d} .
$$

Proof. Since $\mathcal{B}_{r}\left(x^{*}\right) \subset \mathcal{D}$ whenever $r=\operatorname{dist}\left(x^{*}, \partial \mathcal{D}\right)$, using (3.17) it is immediate that

$$
|\mathcal{D}| \geq\left|\mathcal{B}_{r}\left(x^{*}\right)\right| \geq \omega_{d}\left(\Psi^{-1}\left(\frac{\left\|V^{-}\right\|_{\infty}-\inf _{\mathcal{D}} V^{+}+\lambda^{V, \mathcal{D}}}{\theta}\right)\right)^{-d / 2} .
$$

The Faber-Krahn inequality has been previously known only for the classical case $\Psi(u)=u[21$, Th. 1.1], and for the fractional case $\Psi(u)=u^{1 / 2}$.

Remark 3.9 (Torsion). Recall the notation $H_{0}=\Psi(-\Delta)$, and consider the non-local Dirichlet problem

$$
\left\{\begin{aligned}
H_{0}^{\mathcal{D}} v & =1, \quad \text { in } \mathcal{D} \\
v & =0, \quad \text { in } \mathcal{D}^{c}
\end{aligned}\right.
$$

The function $v$ is called torsion, and recently it has been noticed that its maximizer and the maximizer $x^{*}$ of the principal Dirichlet eigenfunction of $H_{0}$ are located very near to each other, though do not coincide. This puzzling phenomenon has been discussed in [7], see also the references therein. Note that the solution has the immediate probabilistic meaning $v(x)=\mathbb{E}^{x}\left[\tau_{\mathcal{D}}\right]$. It is immediate from Corollaries 3.2-3.3 above that with a constant $C=C(d)>0$, we have

$$
\sup _{\mathcal{D}} v(x) \leq C v\left(x^{*}\right)
$$

A similar result was obtained in [61, Cor. 2] for the case of the classical Laplacian in dimension 2. Moreover, for $\Psi(u)=u$, an estimate similar to (3.34) is also known [66].

\section{Compactly supported potentials}

In this section we consider the eigenvalue problems (1.3)-(1.4) for the special choice of bounded potentials with compact support. In case $V=-v \mathbb{1}_{\mathcal{K}}$ with a bounded set $\mathcal{K} \subset \mathbb{R}^{d}$ with non-empty interior, we say that $V$ is a potential well with coupling constant $v>0$.

Concerning the eigenvalue problem in $L^{2}\left(\mathbb{R}^{d}\right)$, recall that the non-local Schrödinger operator $H=\Psi(-\Delta)+V$ admits a Feynman-Kac representation [36] of an eigenfunction $\varphi$ in the form

$$
e^{-t H} \varphi(x)=e^{\lambda t} \mathbb{E}^{x}\left[e^{-\int_{0}^{t} V\left(X_{s}\right) d s} \varphi\left(X_{t}\right)\right], \quad x \in \mathbb{R}^{d}, t \geq 0 .
$$

For a potential well $-v \mathbb{1}_{\mathcal{K}}$ this becomes specifically

$$
e^{-t H} \varphi(x)=e^{\lambda t} \mathbb{E}^{x}\left[e^{v U_{t}^{\mathcal{K}}(X)} \varphi\left(X_{t}\right)\right],
$$


where

$$
U_{t}^{\mathcal{K}}(X)=\int_{0}^{t} \mathbb{1}_{\mathcal{K}}\left(X_{s}\right) d s
$$

is the occupation measure of the set $\mathcal{K}$ by subordinate Brownian motion $\left(X_{t}\right)_{t \geq 0}$.

For non-local Schrödinger operators $H$ above the semigroup $\left\{T_{t}: t \geq 0\right\}, T_{t}=e^{-t H}$, is welldefined and strongly continuous. For all $t>0$, every $T_{t}$ is a bounded operator on every $L^{p}\left(\mathbb{R}^{d}\right)$ space, $1 \leq p \leq \infty$. The operators $T_{t}: L^{p}\left(\mathbb{R}^{d}\right) \rightarrow L^{p}\left(\mathbb{R}^{d}\right)$ for $1 \leq p \leq \infty, t>0$, and $T_{t}: L^{p}\left(\mathbb{R}^{d}\right) \rightarrow L^{\infty}\left(\mathbb{R}^{d}\right)$ for $1<p \leq \infty, t \geq t_{\mathrm{b}}$, and $T_{t}: L^{1}\left(\mathbb{R}^{d}\right) \rightarrow L^{\infty}\left(\mathbb{R}^{d}\right)$ for $t \geq 2 t_{\mathrm{b}}$ are bounded, with some $t_{\mathrm{b}} \geq 0$. Also, for all $t \geq 2 t_{\mathrm{b}}, T_{t}$ has a bounded measurable kernel $u(t, x, y)$ symmetric in $x$ and $y$, i.e., $T_{t} f(x)=\int_{\mathbb{R}^{d}} u(t, x, y) f(y) d y$, for all $f \in L^{p}\left(\mathbb{R}^{d}\right)$ and $1 \leq p \leq \infty$. For all $t>0$ and $f \in L^{\infty}\left(\mathbb{R}^{d}\right)$, $T_{t} f$ is a bounded continuous function. Thus the eigenfunctions solving (1.4) are bounded and continuous, whenever they exist. Also, they have a pointwise decay to zero at infinity. For a subclass of subordinate Brownian motions it is known that the eigenfunctions decay at a rate determined by the Lévy density of $\left(X_{t}\right)_{t \geq 0}$. For further details we refer to [44].

Since these potentials are relatively compact perturbations of $H_{0}=\Psi(-\Delta)$, the essential spectrum is preserved, and thus we have $\operatorname{Spec} H=\mathrm{Spec}_{\text {ess }} H \cup \mathrm{Spec}_{\mathrm{d}} H$, with $\mathrm{Spec}_{\mathrm{ess}} H=\mathrm{Spec}_{\text {ess }} H_{0}=$ $[0, \infty)$. The existence of a discrete component depends on further details of the operator. Generally, $\operatorname{Spec}_{\mathrm{d}} H \subset(-v, 0)$, and $\mathrm{Spec}_{\mathrm{d}} H$ consists of at most a countable set of isolated eigenvalues of finite multiplicity whenever it is non-empty, with possible accumulation point up to zero. For non-negative compactly supported potentials it is known that $\operatorname{Spec}_{\mathrm{d}} H \neq \emptyset$ if $\left(X_{t}\right)_{t \geq 0}$ is a recurrent process [22]. For potential wells this means that at least an eigenfunction exists for every $v>0$ when $\left(X_{t}\right)_{t \geq 0}$ recurrent, on the other hand, it is also possible to show that for transient processes eigenfunctions do not exist if $v$ is too small, but there is at least one if $v$ is large enough.

For the remainder of this section we assume that an eigenfunction exists in either case (1.3)-(1.4), which is thus bounded and continuous. The following result applies for both eigenvalue problems.

Theorem 4.1. Let $\mathcal{D}$ be $\mathbb{R}^{d}$ or a bounded subset of $\mathbb{R}^{d}, V$ be a convex increasing function attaining a global minimum at $\hat{x} \in \mathcal{D}$, and consider the respective eigenvalue equations with a pair $\lambda$ and $\varphi$ such that $\lim _{x \rightarrow z \in \partial \mathcal{D}} \varphi(x)=0$. Furthermore, let $x^{*}$ be the location of a global maximum of $|\varphi|$, and consider the set

$$
\mathcal{U}_{\lambda}=\{x \in \mathcal{D}: V(x) \leq \lambda\} \cap \mathcal{D} .
$$

Then we have $x^{*} \in \mathcal{U}_{\lambda}$ and hence,

$$
\operatorname{dist}\left(x^{*}, \hat{x}\right) \leq \max _{z \in \partial \mathcal{U}_{\lambda}} \operatorname{dist}\left(x^{*}, z\right) .
$$

Proof. Note that we only need to show that $x^{*} \in \mathcal{U}_{\lambda}$. Assume, to the contrary, that $x^{*} \in \mathcal{D} \cap \mathcal{U}_{\lambda}^{c}$. Clearly, we have $V>\lambda$ on $\mathcal{D} \cap \mathcal{U}_{\lambda}^{c}$. Also, we may assume that $\varphi\left(x^{*}\right)>0$. Using the strong Markov property in the Feynman-Kac representation, we find that

$$
\varphi\left(x^{*}\right)=\mathbb{E}^{x^{*}}\left[e^{-\int_{0}^{t \wedge \tau_{\mathcal{u}_{\lambda}^{c}}^{c}}\left(V\left(X_{s}\right)-\lambda\right) \mathrm{d} s} \varphi\left(X_{t \wedge \tau_{\mathcal{U}_{\lambda}}}\right) \mathbb{1}_{\left\{t \wedge \tau_{\mathcal{U}_{\lambda}^{c}}<\tau_{\mathcal{D}}\right\}}\right]
$$

which implies,

$$
1 \leq \mathbb{E}^{x^{*}}\left[e^{-\int_{0}^{t \wedge \tau_{u_{\lambda}}}\left(V\left(X_{s}\right)-\lambda\right) \mathrm{d} s} \mathbb{1}_{\left\{t \wedge \tau_{\left.\mathcal{u}_{\lambda}<\tau_{\mathcal{D}}\right\}}\right.}\right], \quad \forall t>0 .
$$

However, the above is not possible and hence this is a contradiction.

We note that in [6] a related question has been addressed for classical Dirichlet-Schrödinger equations in convex planar domains.

Remark 4.1. It should noted that the convexity of $V$ is not used to find the location of the maximizer. For instance, if we have $V$ compactly supported inside $\mathcal{D}$ and $\lambda<0$, then the same proof above shows that $x^{*} \in \operatorname{supp} V$. 
Next we consider a situation in a bounded domain. The following result shows how far from the support of a not sufficiently negative potential inside a bounded domain a maximizer can move out. This may be compared with Theorem 3.2, in particular, it will be seen that the effect of the potential is exercised by the eigenvalue alone. We will use the following condition on $\Psi$ repeatedly, which we single out here.

Assumption 4.1. Let $\Psi \in \mathcal{B}_{0}$. We assume that for every $\gamma_{0}>0$

$$
\lim _{s \rightarrow 0} \sup _{\gamma \in\left[\gamma_{0}, \infty\right)} \frac{\Psi(s \gamma)}{\Psi(\gamma)}=0
$$

holds.

We will comment and give some examples of Bernstein functions satisfying this assumption following the proof of our next main result.

To explain our next result, consider a potential $V$ compactly supported in $\mathcal{D}$. Note that the lower bound in (3.17) uses the $L^{\infty}$ norm of $V$ and therefore it is difficult to say how the size of the support of $V$ influences the location $x^{*}$. In the next result we make an attempt in this direction. In particular, we show that if $x^{*}$ stays for some reason sufficiently far from $\operatorname{supp} V$, then the lower bound in (3.17) improves. While the assumption may not be easily verifiable at this stage, we find the conclusion interesting as it highlights a mechanism of the delicate balance phenomenon driving the maximizer $x^{*}$ to stabilise.

Theorem 4.2. Let $\Psi$ satisfy Assumption 4.1, and $V$ be a potential with compact support $\operatorname{supp} V=$ $\mathcal{K}$. Consider a convex bounded domain $\mathcal{D} \subset \mathbb{R}^{d}$, containing $\mathcal{K}$, and let $\operatorname{dist}(\mathcal{K}, \partial \mathcal{D})=\kappa>0$. Also, let $\varphi$ be an eigenfunction at eigenvalue $\lambda>0$ solving (1.3), and suppose it is known about a global maximizer $x^{*}$ of $|\varphi|$ that $\operatorname{dist}\left(x^{*}, \partial \mathcal{D}\right) \leq \kappa / 2$. Then there exists a constant $\zeta>0$, dependent on $d$, $\kappa$ and $\Psi$, but not on $\mathcal{D}, \mathcal{K}, \varphi$ or $\lambda$, such that

$$
\operatorname{dist}\left(x^{*}, \partial \mathcal{D}\right) \geq \frac{1}{\sqrt{\Psi^{-1}\left(\frac{\lambda}{\zeta}\right)}} .
$$

Proof. Denote $r=\operatorname{dist}\left(x^{*}, \partial \mathcal{D}\right)$, and without loss of generality assume that $x^{*}=0$. Let $t=\frac{c}{\Psi\left(r^{-2}\right)}$, where the constant $c$ will be chosen below. From the proof of Theorem 3.2 it follows that we can choose $c$ large enough to satisfy

$$
\mathbb{P}_{S}\left(S_{t}^{\Psi}<r^{2}\right)<\frac{1}{4}, \quad \forall r>0 .
$$

Fix this choice of $c$ and define $T_{c}=\frac{c}{\Psi\left(4 \kappa^{-2}\right)}$. Since $r \leq \frac{\kappa}{2}$, we have $t \leq T_{c}$. Using (4.1) we show below that there exists $T_{\circ}>1$ such that

$$
\mathbb{P}_{S}\left(S_{t}^{\Psi} \leq r^{2} T_{\circ}\right) \geq \frac{1}{2}, \text { for all } 0<r<\frac{\kappa}{2} .
$$

Define $Y_{r}=\frac{1}{r^{2}} S_{t}^{\Psi}$. Then the Laplace transform of $Y_{r}$ is given by

$$
\breve{f}(s)=\mathbb{E}\left[e^{-s Y_{r}}\right]=\mathbb{E}\left[e^{-\frac{s}{r^{2}} S_{t}^{\Psi}}\right]=e^{-\frac{c}{\Psi\left(r^{-2}\right)} \Psi\left(s r^{-2}\right)} .
$$

Since $r<\kappa / 2$, using (4.1) and (4.4) we see that $\breve{f}(s) \rightarrow 1$ as $s \rightarrow 0$, uniformly in $r \in\left(0, \frac{\kappa}{2}\right]$. Thus by the uniform Tauberian theorem [52, Th. 3], we obtain

$$
\mathbb{P}_{S}\left(Y_{r} \leq y\right) \rightarrow 1 \quad \text { as } y \rightarrow \infty, \quad \text { uniformly in } r \in\left(0, \frac{\kappa}{2}\right] \text {. }
$$

Hence we can find $T_{\circ}>1$ satisfying

$$
\mathbb{P}_{S}\left(S_{t}^{\Psi} \leq r^{2} T_{\circ}\right) \geq \frac{1}{2}, \quad \text { for all } 0<r<\frac{\kappa}{2} .
$$


Combining (4.3) and (4.5) we obtain that

$$
\mathbb{P}_{S}\left(S_{t}^{\Psi} \in\left[r^{2}, r^{2} T_{\circ}\right]\right)>\frac{1}{4}, \quad \forall r \in\left(0, \frac{\kappa}{2}\right] .
$$

Now we fix the above choice of $T_{\circ}$, which depends on $c, \kappa$ and $\Psi$. On the other hand, since $\mathcal{D}$ is convex, we may assume that there exists a point $z_{0} \in \partial \mathcal{D}$ such that $\operatorname{dist}\left(0, z_{0}\right)=r, z_{0}$ lies on the $x_{1}$-axis and $\mathcal{D}$ lies of the on the complement of the half-space $\left\{y \in \mathbb{R}^{d}: z_{0} \cdot y \geq r^{2}\right\}$. Define $\chi:\left[0, T_{\circ}\right] \rightarrow \mathbb{R}^{d}$ by

$$
\chi(s)=2 \sqrt{s} e_{1}
$$

where $e_{1}$ is the unit vector along the $x_{1}$-axis. Note that $\operatorname{dist}\left(z_{0}, \chi\left(r^{2}\right)\right)=r$ and $z_{0} \cdot \chi\left(r^{2}\right)=2 r^{2}$. Define for $\delta \in\left(0, \frac{\kappa}{4} \wedge \frac{1}{2}\right)$

$$
N_{\delta}=\left\{f \in \mathcal{C}\left(\left[0, T_{\circ}\right], \mathbb{R}^{d}\right): f(0)=0 \text { and } \max _{s \in\left[0, T_{\circ}\right]}|f(s)-\chi(s)|<\delta\right\},
$$

i.e., a $\delta$-neighbourhood of $\chi$ in $\mathcal{C}_{0}\left(\left[0, T_{\circ}\right], \mathbb{R}^{d}\right)$, the space of $\mathbb{R}^{d}$-valued continuous functions on $\left[0, T_{\circ}\right]$ with value 0 at $s=0$. By the Stroock-Varadhan support theorem it follows that there exists $\delta_{1}>0$ such that

$$
\mathbb{P}_{W}^{0}\left(\frac{1}{r} B_{r^{2} s} \in N_{\delta}\right)=\mathbb{P}_{W}^{0}\left(B_{s} \in N_{\delta}\right)=\delta_{1}>0
$$

Note the equivalence of the events

$$
\left\{\max _{s \in\left[0, T_{\circ}\right]}\left|\frac{1}{r} B_{r^{2} s}-\chi(s)\right|<\delta\right\}=\left\{\max _{s \in\left[0, T_{\circ}\right]}\left|B_{r^{2} s}-r \chi(s)\right|<r \delta\right\}=\left\{\max _{s \in\left[0, T_{\circ} r^{2}\right]}\left|B_{s}-\chi(s)\right|<r \delta\right\},
$$

where in the last equality we used that $r \chi(s)=\chi\left(r^{2} s\right)$. Thus we find

$$
\mathbb{P}_{W}^{0}\left(\max _{s \in\left[0, r^{2} T_{\circ}\right]}\left|B_{s}-\chi(s)\right|<r \delta\right)=\delta_{1} .
$$

Combining (4.6) and (4.8) we have

$$
\begin{aligned}
& \mathbb{P}^{0}\left((\omega, \varpi): \sup _{s \in[0, t]}\left|B_{S_{s}^{\Psi}}-\chi\left(S_{s}^{\Psi}\right)\right|<r \delta, S_{t}^{\Psi} \in\left[r^{2}, r^{2} T_{\circ}\right]\right) \\
& \geq \mathbb{P}^{0}\left((\omega, \varpi): \max _{s \in\left[0, r^{2} T_{\circ}\right]}\left|B_{s}(\omega)-\chi(s)\right|<r \delta, S_{t}^{\Psi}(\varpi) \in\left[r^{2}, r^{2} T_{\circ}\right]\right) \\
& \quad=\mathbb{P}_{W}^{0}\left(\max _{s \in\left[0, r^{2} T_{\circ}\right]}\left|B_{s}-\chi(s)\right|<r \delta\right) \mathbb{P}_{S}\left(S_{t}^{\Psi} \in\left[r^{2}, r^{2} T_{\circ}\right]\right) \geq \frac{\delta_{1}}{4},
\end{aligned}
$$

where the third line follows from the independence of Brownian motion and the subordinator. By the construction of $\chi$ it is seen that every path satisfying

$$
\sup _{s \in[0, t]}\left|B_{S_{s}^{\Psi}}-\chi\left(S_{s}^{\Psi}\right)\right|<r \delta, \quad S_{t}^{\Psi} \in\left[r^{2}, r^{2} T_{\circ}\right],
$$

must leave $\mathcal{D}$ by time $t$ since $B_{S_{t}^{\Psi}} \in \mathcal{D}^{c}$, and it does not enter $\mathcal{K}$ in the time interval $[0, t]$. Thus by (4.9) we obtain

$$
\mathbb{P}^{0}\left(\tau_{\mathcal{D}} \leq t \wedge \tau_{\mathcal{K}^{c}}\right)>\frac{\delta_{1}}{4}
$$

Then by the Feynman-Kac formula and the strong Markov property it follows that

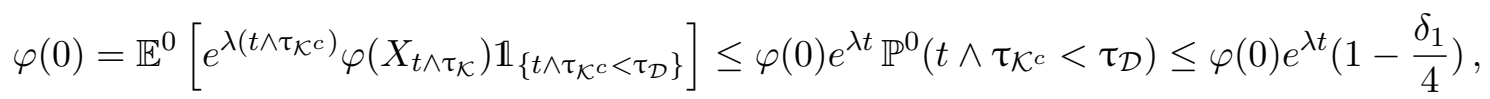

using (4.10). By taking logarithms both sides, we obtain (4.2).

There is a large family of subordinate Brownian motions satisfying Assumption 4.1. First we show a general statement and then illustrate it by some important examples. 
Lemma 4.1. Suppose that $\Psi$ is unbounded and regularly varying at infinity, i.e., with a slowly varying function $\ell$ and constant $\beta>0$ we have

$$
\Psi(u) \asymp u^{\beta} \ell(\beta), \quad \text { for all large } u .
$$

Then Assumption 4.1 holds.

Proof. It suffices to show that for any sequence $\left(s_{n}, \gamma_{n}\right)$ with

$$
s_{n} \rightarrow 0, \quad \gamma_{n} \rightarrow \infty, \quad \text { and } s_{n} \gamma_{n} \rightarrow \infty,
$$

we have

$$
\lim _{n \rightarrow \infty} \frac{\Psi\left(s_{n} \gamma_{n}\right)}{\Psi\left(\gamma_{n}\right)}=0
$$

Fix any $\varepsilon>0$. Then for large $n$,

$$
\frac{\Psi\left(s_{n} \gamma_{n}\right)}{\Psi\left(\gamma_{n}\right)} \leq \frac{\Psi\left(\varepsilon \gamma_{n}\right)}{\Psi\left(\gamma_{n}\right)} \asymp \varepsilon^{\beta} \frac{\ell\left(\varepsilon \gamma_{n}\right)}{\ell\left(\gamma_{n}\right)} \asymp \varepsilon^{\beta} .
$$

Hence (4.11) follows.

Example 4.1. By Lemma 4.1 the following Bernstein functions satisfy Assumption 4.1:

(i) $\Psi(u)=u^{\alpha / 2}, \alpha \in(0,2]$.

(ii) $\Psi(u)=\left(u+m^{2 / \alpha}\right)^{\alpha / 2}-m, m>0, \alpha \in(0,2)$.

(iii) $\Psi(u)=u^{\alpha / 2}+u^{\beta / 2}, 0<\beta<\alpha \in(0,2]$.

(iv) $\Psi(u)=u^{\alpha / 2}(\log (1+u))^{\beta / 2}, \alpha \in(0,2), \beta \in(0,2-\alpha)$.

(v) $\Psi(u)=u^{\alpha / 2}(\log (1+u))^{-\beta / 2}, \alpha \in(0,2], \beta \in[0, \alpha)$.

Example 4.2. On the other hand, $\Psi(u)=\log \left(1+u^{\alpha / 2}\right), \alpha \in(0,2]$, does not satisfy Assumption 4.1. To see this note that for $s=\frac{1}{n}$ and $\gamma=n^{2}$ we have

$$
\lim _{n \rightarrow \infty} \frac{\Psi(s \gamma)}{\Psi(\gamma)}=\lim _{n \rightarrow \infty} \frac{\log \left(1+n^{\alpha / 2}\right)}{\log \left(1+n^{\alpha}\right)} \geq \frac{1}{2} .
$$

In the remaining part of this section we consider the eigenvalue problem in full space.

Theorem 4.3. Consider the operator $H$ given by (1.2), $\operatorname{supp} V=\mathcal{K}$, and let $\varphi$ be a solution of the Schrödinger eigenvalue problem (1.4) for $H$, corresponding to eigenvalue $\lambda=-|\lambda|<0$. If $|\varphi|$ has a global maximum at $x^{*} \in \mathbb{R}^{d}$, then $x^{*} \in \mathcal{K}$.

Proof. We show that there is no maximizer in $\mathcal{K}^{c}$. Assume, to the contrary, that $x^{*} \in \mathcal{K}$. Therefore, for a suitable $\delta>0$ we have $\mathcal{B}_{\delta}\left(x^{*}\right) \in \mathcal{K}^{c}$. Let $\tau_{\delta}$ be the exit time from the ball $\mathcal{B}_{\delta}\left(x^{*}\right)$. Since $\mathbb{E}^{x^{*}}\left[\tau_{\delta}\right]>0$, we find $t>0$ such that $\mathbb{P}^{x^{*}}\left(\tau_{\delta}>t\right)>0$. As before, we can also assume that $\varphi\left(x^{*}\right)>0$. By the Feynman-Kac representation we have

$$
\begin{aligned}
\varphi\left(x^{*}\right) & =\mathbb{E}^{x^{*}}\left[e^{\lambda\left(t \wedge \tau_{\delta}\right)} \varphi\left(X_{t \wedge \tau_{\delta}}\right)\right] \\
& \leq e^{\lambda t} \mathbb{E}^{x^{*}}\left[\varphi\left(X_{t}\right) \mathbb{1}_{\left\{\tau_{\delta}>t\right\}}\right]+\left[e^{\lambda \tau_{\delta}} \varphi\left(X_{t}\right) \mathbb{1}_{\left\{\tau_{\delta} \leq t\right\}}\right] \\
& \leq e^{\lambda t} \varphi\left(x^{*}\right) \mathbb{P}^{x^{*}}\left(\tau_{\delta}>t\right)+\varphi\left(x^{*}\right) \mathbb{P}^{x^{*}}\left(\tau_{\delta} \leq t\right) .
\end{aligned}
$$

This would imply $e^{\lambda t}>1$, which is a contradiction as $\lambda<0$. Hence $x^{*} \in \mathcal{K}$.

Remark 4.2. Recall that the eigenfunctions are continuous, as mentioned earlier. Since $V$ is bounded, from the Feynman-Kac representation we have for every $t>0$ that

$$
|\varphi(x)| \leq e^{\left(\|V\|_{\infty}-\lambda\right) t} \mathbb{E}^{x}\left[|\varphi|^{2}\left(X_{t}\right)\right]^{1 / 2} \leq e^{\left(\|V\|_{\infty}-\lambda_{0}\right) t}\left(\int_{\mathbb{R}^{d}}|\varphi(y)|^{2} q_{t}(x-y) \mathrm{d} y\right)^{1 / 2}
$$


where $q_{t}(x, y)=q_{t}(x-y)$ denotes the transition density of $\left(X_{t}\right)_{t \geq 0}$ starting at $X_{0}=x$. It follows by subordination, see $(2.4)$, that

$$
q_{t}(x-y)=\int_{0}^{\infty} \frac{1}{(4 \pi s)^{d / 2}} e^{-\frac{|x-y|^{2}}{4 s}} \mathbb{P}_{S}\left(S_{t}^{\Psi} \in \mathrm{d} s\right) .
$$

Therefore, for every fixed $y$ we have $q_{t}(x-y) \rightarrow 0$ as $|x| \rightarrow \infty$. Moreover, if $\Psi$ satisfies the Hartman-Wintner condition (3.4), then $q_{t}(x, y)$ is bounded and continuous. Hence by dominated convergence we obtain from (4.12) that $\lim _{|x| \rightarrow \infty}|\varphi(x)|=0$, thus every eigenfunction attains its maximum in $\mathbb{R}^{d}$.

Finally, we show how deep inside the support the maximizer can be for a potential well. We denote by Int $\mathcal{K}$ the interior of $\mathcal{K}$.

Theorem 4.4. Let $V=-v \mathbb{1}_{\mathcal{K}}$ with a bounded convex set $\mathcal{K}$, and $\varphi$ be an eigenfunction corresponding to eigenvalue $\lambda=-|\lambda|<0$ solving the eigenvalue problem (1.4). Suppose that $\Psi$ is unbounded and satisfies Assumption 4.1. Then there exist two constants $\varrho_{1}, \varrho_{2}>0$, dependent only on $\Psi$ and $\operatorname{inrad} \mathcal{K}$, such that if

$$
\frac{v-|\lambda|}{|\lambda|} \leq \varrho_{1}
$$

then $x^{*} \in \operatorname{Int} \mathcal{K}$ and

$$
\operatorname{dist}\left(x^{*}, \partial \mathcal{K}\right) \geq \frac{1}{\sqrt{\Psi^{-1}\left(\frac{|\lambda|}{\varrho_{2}}\right)}} .
$$

Proof. Step 1: First we prove (4.14) assuming that $x^{*} \in$ Int $\mathcal{K}$. By a shift we can assume that $x^{*}=0$ with no loss of generality, and we denote $r=\operatorname{dist}\left(x^{*}, \partial \mathcal{K}\right)>0$. Let $t=\frac{c}{\Psi\left(r^{-2}\right)}$ where the constant $c$ will be chosen later. From the proof of Theorem 4.2 we see that we can choose $c$ large enough such that

$$
\mathbb{P}_{S}\left(S_{t}^{\Psi} \in\left[r^{2}, r^{2} T_{0}\right]\right)=\delta_{1}>\frac{1}{4}, \quad \forall r \in(0, \operatorname{inrad} \mathcal{K}]
$$

see (4.6) above. Therefore, by the independence of increments we have from (4.15) that

$$
\mathbb{P}_{S}\left(S_{t}^{\Psi} \in\left[r^{2}, r^{2} T_{\circ}\right], S_{2 t}^{\Psi}-S_{t}^{\Psi} \in\left[r^{2}, r^{2} T_{\circ}\right]\right)=\delta_{1}^{2}, \quad \forall 0<r \leq \operatorname{inrad} \mathcal{K} .
$$

Now we fix the above choice of $T_{\circ}$ which depends on $c$ and $\Psi$ and inrad $\mathcal{K}$ (recall that $r$ and $t$ are related). Since $\mathcal{K}$ is convex, we may assume that the point $z_{0}=(r, 0, \ldots, 0) \in \partial \mathcal{K}$ is such that $\operatorname{dist}\left(0, z_{0}\right)=r, \mathcal{K}$ lies on the on the complement of the half-space $\left\{y \in \mathbb{R}^{d}: z_{0} \cdot y \geq r^{2}\right\}$. Define $\chi:\left[0, T_{\circ}\right] \rightarrow \mathbb{R}^{d}$ by

$$
\chi(s)=2 s,
$$

Note that $\operatorname{dist}\left(0, \chi\left(\frac{1}{2} r\right)\right)=r$. Define

$$
\mathcal{N}=\left\{f \in \mathcal{C}\left(\left[0,2 T_{\circ}\right], \mathbb{R}\right): f(0)=0 \text { and } \max _{s \in\left[0,2 T_{\circ}\right]}|f(s)-\chi(s)|<\frac{1}{2}\right\} .
$$

In a similar manner as in the proof of Theorem 4.2 we find that there is a $\delta_{2}>0$ such that

$$
\mathbb{P}_{W}^{0}\left(\frac{1}{r} B_{r^{2} .} \in \mathcal{N}\right)=\mathbb{P}_{W}^{0}\left(B^{1} \in \mathcal{N}\right)=\delta_{2} .
$$

Also, we have

$$
\left\{\max _{s \in\left[0,2 T_{\circ}\right]}\left|\frac{1}{r} B_{r^{2} s}^{1}-\chi(s)\right|<\frac{1}{2}\right\}=\left\{\max _{s \in\left[0,2 r^{2} T_{\circ}\right]}\left|B_{s}^{1}-\frac{1}{r} \chi(s)\right|<\frac{r}{2}\right\},
$$


using scaling and that $r \chi(s)=\frac{1}{r} \chi\left(r^{2} s\right)$. Thus we obtain

$$
\mathbb{P}_{W}^{0}\left(\max _{s \in\left[0,2 r^{2} T_{\circ}\right]}\left|B_{s}^{1}-\frac{1}{r} \chi(s)\right|<\frac{r}{2}\right)=\delta_{2} .
$$

Combining (4.16) and (4.18) gives

$$
\begin{aligned}
& \mathbb{P}^{0}\left((\omega, \varpi): \max _{s \in\left[0,2 T_{\circ} r^{2}\right]}\left|B_{s}^{1}(\omega)-\frac{1}{r} \chi(s)\right|<r / 2, S_{t}^{\Psi}(\varpi) \in\left[r^{2}, r^{2} T_{\circ}\right], S_{2 t}^{\Psi}(\varpi)-S_{t}^{\Psi}(\varpi) \in\left[r^{2}, r^{2} T_{\circ}\right]\right) \\
& =\mathbb{P}_{W}^{0}\left(\max _{s \in\left[0,2 T_{\circ} r^{2}\right]}\left|B_{s}^{1}-\chi(s)\right|<r / 2\right) \mathbb{P}_{S}\left(S_{t}^{\Psi} \in\left[r^{2}, r^{2} T_{\circ}\right], S_{2 t}^{\Psi}-S_{t}^{\Psi} \in\left[r^{2}, r^{2} T_{\circ}\right]\right)=\delta_{2} \delta_{1}^{2},
\end{aligned}
$$

where the third line follows from the independence of the two processes. Let

$\widehat{\Omega}=\left\{(\omega, \varpi): \max _{s \in\left[0,2 T_{\circ} r^{2}\right]}\left|B_{s}^{1}(\omega)-\frac{1}{r} \chi(s)\right|<\frac{r}{2}, \quad S_{t}^{\Psi}(\varpi) \in\left[r^{2}, r^{2} T_{\circ}\right], \quad S_{2 t}^{\Psi}(\varpi)-S_{t}^{\Psi}(\varpi) \in\left[r^{2}, r^{2} T_{\circ}\right]\right\}$.

We see that

$$
\widehat{\Omega} \subset\left\{(\omega, \varpi): \sup _{s \in[0, t]}\left|B_{S_{s}^{\Psi}}^{1}-\frac{1}{r} \chi\left(S_{s}^{\Psi}\right)\right|<\frac{r}{2}, \quad S_{t}^{\Psi} \in\left[r^{2}, r^{2} T_{\circ}\right], \quad S_{2 t}^{\Psi}-S_{t}^{\Psi} \in\left[r^{2}, r^{2} T_{\circ}\right]\right\} .
$$

By the construction of $\chi$ it follows that for every $(\omega, \varpi) \in \widehat{\Omega}, B_{S_{t}^{\Psi}} \in \mathcal{K}^{c}$ and the paths of $B_{S_{s}^{\Psi}}$ stay in $\mathcal{K}^{c}$ for all $s \in[t, 2 t]$. This observation will play a key role in our analysis below.

Let $\delta=\delta_{2} \delta_{1}^{2}$, and define

$$
2 \varrho_{1}=\frac{\delta}{2-\delta} \in(0,1)
$$

and a function $\xi: \mathbb{R} \rightarrow \mathbb{R}^{+}$by

$$
\xi(y)=\delta e^{-\frac{1}{2}\left(1-\varrho_{1}\right) y}+(1-\delta) e^{\varrho_{1} y} .
$$

It is direct to see that $\xi^{\prime}\left(\varepsilon_{0}\right)=0$ gives

$$
\varepsilon_{0}=\frac{2}{1+\varrho_{1}} \log \frac{\delta\left(1-\varrho_{1}\right)}{2 \varrho_{1}(1-\delta)} .
$$

Since

$$
\varrho_{1}<\frac{\delta}{2-\delta} \quad \text { implies } \frac{\delta\left(1-\varrho_{1}\right)}{2 \varrho_{1}(1-\delta)}>1,
$$

we have $\varepsilon_{0}>0$. Again observe that $\xi^{\prime}(0)<0$, and therefore $\xi(y)<1$ for $y \in\left(0, \varepsilon_{0}\right)$.

Suppose now that $\frac{v-|\lambda|}{|\lambda|} \leq \varrho_{1}$. By the Feyman-Kac representation we have

$$
\varphi(0)=\mathbb{E}^{0}\left[e^{\int_{0}^{2 t}\left(\lambda-V\left(X_{s}\right)\right) \mathrm{d} s} \varphi\left(X_{2 t}\right)\right]
$$

which, in turn, implies

$$
\begin{aligned}
1 & \leq \mathbb{E}^{0}\left[e^{\int_{0}^{2 t}\left(\lambda-V\left(X_{s}\right)\right) \mathrm{d} s}\right] \\
& =\mathbb{E}^{0}\left[e^{\int_{0}^{2 t}\left(\lambda-V\left(X_{s}\right)\right) \mathrm{d} s} \mathbb{1}_{\widehat{\Omega}}\right]+\mathbb{E}^{0}\left[e^{\int_{0}^{2 t}\left(\lambda-V\left(X_{s}\right)\right) \mathrm{d} s} \mathbb{1}_{\widehat{\Omega}^{c}}\right] \\
& \leq \mathbb{E}^{0}\left[e^{\int_{0}^{2 t}\left(\lambda-V\left(X_{s}\right)\right) \mathrm{d} s+\lambda t} \mathbb{1}_{\widehat{\Omega}}\right]+(1-\delta) e^{(v+\lambda) 2 t} \\
& \leq \delta e^{(v+\lambda) t-|\lambda| t}+(1-\delta) e^{(v+\lambda) 2 t} \leq \delta e^{\varrho_{1}|\lambda| t-|\lambda| t}+(1-\delta) e^{\varrho_{1}|\lambda| 2 t}=\xi(2 t|\lambda|),
\end{aligned}
$$

where in the fourth line we used (4.19). Since $2 t|\lambda|>0$ and $\xi(2 t|\lambda|) \geq 1$, we conclude that

$$
2 t|\lambda| \geq \varepsilon_{0}
$$

holds. Hence (4.14) follows with $\varrho_{2}=\frac{\varepsilon_{0}}{2 c}$. 
Step 2: To conclude, we prove that under the condition (4.13) we have $x^{*} \notin \partial \mathcal{K}$. Like before, we may assume that $x^{*}=0$ and $\mathcal{K} \subset\left\{x_{1} \leq 0\right\}$. Note that the estimate (4.19) holds uniformly in $r \in(0, \operatorname{inrad} \mathcal{K})$. Since 0 is on the boundary of $\mathcal{K}$ and the function $\chi$, defined above, lies in $\left\{x_{1} \geq 0\right\}$, we observe that for every $r>0$ and every $(\omega, \varpi) \in \widehat{\Omega}=\widehat{\Omega}_{r}$ we have $B_{S_{t}^{\Psi}} \in \mathcal{K}^{c}$ and the paths $B_{S_{s}^{\Psi}}$ in stay $\mathcal{K}^{c}$ for $s \in[t, 2 t]$, where $t=\frac{c}{\Psi\left(r^{-2}\right)}$ and $c$ is chosen the same as before. Therefore, following a similar argument as in the proof of (4.20), we obtain

$$
1 \leq \xi(2 t|\lambda|)
$$

for all $r>0$. Since $t \rightarrow 0$ as $r \rightarrow 0$, and since $\Psi$ is unbounded, the above estimate cannot hold for small $t$. Thus we have a contradiction showing that $0=x^{*} \in \operatorname{Int} \mathcal{K}$.

Remark 4.3. We note that for a potential well $V=-v \mathbb{1}_{\mathcal{K}}, v>0$, we have

$$
v-|\lambda| \leq \lambda_{1}^{\mathcal{K}},
$$

where $\lambda_{1}^{\mathcal{K}}$ is the principal eigenvalue of $\Psi(-\Delta)$ in $\mathcal{K}$ with Dirichlet exterior condition on $\mathcal{K}^{c}$. Indeed, from the Feynman-Kac formula we get that

$$
\begin{aligned}
\varphi(x) & \geq \mathbb{E}^{x}\left[e^{\int_{0}^{t}\left(v \mathbb{1}_{\mathcal{K}}\left(X_{s}\right)+\lambda\right) \mathrm{d} s} \varphi\left(X_{t}\right) \mathbb{1}_{\left\{t<\tau_{\mathcal{K}}\right\}}\right] \\
& \geq e^{t(v-|\lambda|)} \min _{y \in \mathcal{K}} \varphi(y) \mathbb{P}^{x}\left(t<\tau_{\mathcal{K}}\right), \quad x \in \mathcal{K} .
\end{aligned}
$$

By taking logarithms on both sides and dividing by $t>0$, we get

$$
v-|\lambda| \leq-\limsup _{t \rightarrow \infty} \frac{1}{t} \log \mathbb{P}^{x}\left(t<\tau_{\mathcal{K}}\right) \leq \lambda_{1}^{\mathcal{K}} .
$$

Thus the numerator at the left hand side of (4.13) is always bounded by $\lambda_{1}^{\mathcal{K}}$, and so for $|\lambda|$ large enough (4.13) holds. Also, notice that the result in Theorem 4.4 continues to hold for more general potentials $V$ supported on $\mathcal{K}$ and $\lambda<0$. In this situation (4.13) will be replaced by

$$
\frac{-\min _{x \in \mathcal{K}} V(x)-|\lambda|}{|\lambda|} \leq \varrho_{1} .
$$

Notice that the dependence of $\varrho_{1}$ and $\varrho_{2}$ on inrad $\mathcal{K}$ comes from (4.1), which has been crucially used in (4.5). This dependence can be waived for a class of $\Psi$ for which (4.1) holds uniformly in $\gamma_{0}>0$, i.e., when

$$
\lim _{s \rightarrow 0} \sup _{\gamma \in(0, \infty)} \frac{\Psi(s \gamma)}{\Psi(\gamma)}=0 .
$$

Observe that if $\Psi$ satisfies Assumption 3.1, then (4.21) holds. Indeed, we have then

$$
\lim _{s \rightarrow 0} \sup _{\gamma \in(0, \infty)} \frac{\Psi(s \gamma)}{\Psi(\gamma)}=\lim _{s \rightarrow 0} \sup _{\gamma \in(0, \infty)} \frac{\Psi(s \gamma)}{\Psi\left(s^{-1} s \gamma\right)} \lesssim \lim _{s \rightarrow 0} s^{\mu}=0 .
$$

Moreover, (4.5)-(4.6) follow then uniformly in $r \in(0, \infty)$. Therefore, in this case $\varrho_{1}$ and $\varrho_{2}$ only depend on $\Psi$ and not on inrad $\mathcal{K}$. This is recorded in the following result.

Theorem 4.5. Suppose that $\Psi$ satisfies Assumption 3.1, and let $\varphi$ and $\lambda=-|\lambda|$ solve the eigenvalue equation (1.4) for $H$ with a potential well $V=-v \mathbb{1}_{\mathcal{K}}$. Then there exist positive $\varrho_{1}, \varrho_{2}$, dependent only on $\Psi$, such that if

$$
\frac{v-|\lambda|}{|\lambda|} \leq \varrho_{1}
$$

then $x^{*} \in \operatorname{Int} \mathcal{K}$ and

$$
\operatorname{dist}\left(x^{*}, \partial \mathcal{K}\right) \geq \frac{1}{\sqrt{\Psi^{-1}\left(\frac{|\lambda|}{\varrho_{2}}\right)}} .
$$


Theorems 4.4-4.5 have the following interesting "no-go" type consequence.

Corollary 4.1. Under the conditions of Theorems 4.4-4.5 we have that whenever

$$
v<\varrho_{2} \Psi\left(\frac{1}{(\operatorname{inrad} \mathcal{K})^{2}}\right),
$$

then either $|\lambda|<v /\left(1+\varrho_{1}\right)$ or the non-local Schrödinger operator $H$ has no $L^{2}$-eigenfunctions.

Proof. We have trivially $\operatorname{dist}\left(x^{*}, \partial \mathcal{K}\right)<\operatorname{inrad} \mathcal{K}$. Also, $|\lambda|<v$, and $\Psi^{-1}$ is an increasing function. Hence Theorems 4.4-4.5 give

$$
\operatorname{inrad} \mathcal{K} \geq \frac{1}{\sqrt{\Psi^{-1}\left(\frac{v}{\varrho_{2}}\right)}}
$$

implying the result.

\section{Remark 4.4.}

(i) We note that, using direct techniques of differential equations, for usual Schrödinger operators $H=-\Delta-v \mathbb{1}_{\mathcal{B}_{a}}$ in $L^{2}\left(\mathbb{R}^{d}\right)$, it is well-known that for $d \geq 3$, the smallness of the quantity $v a^{2}$ implies that no $L^{2}$-eigenfunctions exist. Using the Birman-Schwinger principle, bounds on $v a^{\alpha}$ can also be derived ruling out $L^{2}$-eigenfunctions of $H=(-\Delta)^{\alpha / 2}-v \mathbb{1}_{\mathcal{B}_{a}}$ and further non-local operators [54]. Although the constants may in general differ, we have the same type of bounds resulting from Corollary 4.1 above.

(ii) We can also use Green functions to find a "no-go" type consequence, which does not involve (4.13). Suppose that $d \geq 3$ and the transition density probability function of $\left(X_{t}\right)_{t \geq 0}$ decays to 0 as $t \rightarrow \infty$. Then the ground state $\varphi_{1}$ of $H=\Psi(-\Delta)-v \mathbb{1}_{\mathcal{K}}$ has the representation

$$
\varphi_{1}(x)=\int_{\mathbb{R}^{d}}\left(\lambda_{1}-V(y)\right) \varphi(y) G(x, y) \mathrm{d} y,
$$

where $G(\cdot, \cdot)$ is the associated Green function. It is known [32, Th. 3] that there exists a constant $C_{d}$, dependent only on $d$, such that

$$
G(x, y) \leq \frac{C_{d}}{|x-y|^{d} \Psi\left(|x-y|^{-2}\right)} .
$$

Let $R=\operatorname{diam} \mathcal{K}$. Since $x^{*} \in \mathcal{K}$, by Theorem 4.3, and $\lambda_{1}<0$ we see from (4.22) that

which implies

$$
\varphi_{1}\left(x^{*}\right) \leq C_{d}\left(v-\left|\lambda_{1}\right|\right) \varphi_{1}\left(x^{*}\right) \int_{\mathcal{K}} \frac{\mathrm{d} y}{\left|x^{*}-y\right|^{d} \Psi\left(\left|x^{*}-y\right|^{-2}\right)},
$$

$$
1 \leq C_{d}\left(v-\left|\lambda_{1}\right|\right) \int_{\mathcal{B}_{R}\left(x^{*}\right)} \frac{\mathrm{d} y}{\left|x^{*}-y\right|^{d} \Psi\left(\left|x^{*}-y\right|^{-2}\right)}=C_{d} d \omega_{d} v \int_{0}^{R} \frac{\mathrm{d} s}{s \Psi\left(s^{-2}\right)},
$$

where $\omega_{d}$ denotes the volume of the unit ball in $\mathbb{R}^{d}$. Therefore, if the right hand side is finite (for example, for $\Psi$ satisfying Assumption 3.1), then there is no ground state whenever

$$
v<\frac{1}{C_{d} d \omega_{d} \int_{0}^{R} \frac{\mathrm{d} s}{s \Psi\left(s^{-2}\right)}} .
$$

Finally we note that our technique in proving Theorem 4.4 is also applicable to a more general class of potentials. Consider equation (1.4). For $V$ convex and increasing we have shown in Theorem 4.1 that the maximizer $x^{*} \in \mathcal{U}_{\lambda}=\{x \in \mathcal{D}: V(x) \leq \lambda\} \cap \mathcal{D}$. For $\delta>0$ we define the $\delta$-neighborhood of $\mathcal{U}_{\lambda}$, i.e.

$$
\mathcal{U}_{\lambda}^{\delta}=\left\{x \in \mathbb{R}^{d}: \operatorname{dist}\left(x, \mathcal{U}_{\lambda}\right) \leq \delta\right\} .
$$

The following result provides a sufficient condition for the maximizer to be strictly inside $\mathcal{U}_{\lambda}$. 
Theorem 4.6. Suppose that $\Psi$ satisfies Assumption 3.1. There exist positive constants $\varrho_{1}$ and $\varrho_{2}$, dependent only on $\Psi$, such that if for some $\delta \in\left(0\right.$, inrad $\left.\mathcal{U}_{\lambda}\right)$

then

$$
\frac{\lambda-\min _{x \in \mathbb{R}^{d}} V(x)}{\min _{x \in \mathbb{R}^{d} \backslash u_{\lambda}^{\delta}}(V(x)-\lambda)} \leq \varrho_{1},
$$

$$
\operatorname{dist}\left(x^{*}, \partial \mathcal{U}_{\lambda}\right) \geq \frac{1}{\sqrt{\Psi^{-1}\left(\frac{\min _{x \in \mathbb{R}^{d} \backslash \mathcal{B}_{\lambda}^{\delta}}(V(x)-\lambda)}{\varrho^{2}}\right)}} .
$$

Acknowledgments: This research of AB was supported in part by an INSPIRE faculty fellowship and a DST-SERB grant EMR/2016/004810. We thank the anonymous referee for a careful reading of the manuscript and helpful suggestions.

\section{REFERENCES}

[1] R. Bañuelos: Four unknown constants, Oberwolfach reports No. 06, 2009

[2] R. Bañuelos and K. Burdzy: On the "hot spots" conjecture of J. Rauch, J. Funct. Anal. 164 (1999), 1-33

[3] R. Bañuelos and T. Carroll: Brownian motion and the fundamental frequency of a drum, Duke Math. J. 75 (1994), 575-602

[4] R. Bañuelos, T. Kulczycki and P.J. Méndez-Hernández: On the shape of the ground state eigenfunction for stable processes, Potential Anal. 24 (2006), 205-221

[5] R. Bañuelos, R. Latała and P.J. Méndez-Hernández: A Brascamp-Lieb-Luttinger-type inequality and applications to symmetric stable processes, Proc. Amer. Math. Soc. 129 (2001), 2997-3008

[6] T. Beck: The shape of the level sets of the first eigenfunction of a class of two-dimensional Schrödinger operators, Trans. Amer. Math. Soc. 370 (2018), 3197-3244

[7] B.A. Benson, R.S. Laugesen, M. Minion and B.A. Siudeja; Torsion and ground state maxima: close but not the same, Irish Math. Soc. Bulletin 78, 2016, 81-88

[8] A. Biswas: Location of maximizers of eigenfunctions of fractional Schrödinger's equation, Math. Phys. Anal. Geom. 20 (2017), 25

[9] A. Biswas and J. Lörinczi: Maximum principles and Aleksandrov-Bakelman-Pucci type estimates for non-local Schrödinger equations with exterior conditions, arXiv:1711.09267, 2017 (submitted for publication)

[10] A. Biswas and J. Lőrinczi: Maximum principles for time-fractional Cauchy problems with spatially non-local components, Fract. Calc. Appl. Anal. 21, 1335-1359, 2018

[11] K. Bogdan and T. Byczkowski: Potential theory for the $\alpha$-stable Schrödinger operator on bounded Lipschitz domains, Studia Math. 133 (1999), 53-92

[12] K. Bogdan and T. Byczkowski: Potential theory of Schrödinger operator based on fractional Laplacian, Probab. Math. Statist. 20 (2000), 293-335

[13] K. Bogdan, T. Grzywny and M. Ryznar: Dirichlet heat kernel for unimodal Lévy processes, Stoch. Proc. Appl. 124 (2014), 3612-3650

[14] K. Bogdan, T. Grzywny and M. Ryznar: Density and tails of unimodal convolution semigroups, J. Funct. Anal. 266 (2014), 3543-3571

[15] K. Bogdan, T. Grzywny and M. Ryznar: Barriers, exit time and survival probability for unimodal Lévy processes, Probab. Theory Relat. Fields 162 (2015), no. 1-2, 155-198

[16] B. Böttcher, R. Schilling, J. Wang: Lévy-Type Processes: Construction, Approximation and Sample Path Properties, Lecture Notes in Mathematics 2099, Lévy Matters vol. III, 2013

[17] L. Brasco, R. Magnanini and P. Salani: The location of the hot spot in a grounded convex conductor, Indiana Univ. Math. J. 60 (2011), no. 2, 633-659 
[18] A. Buades, B. Coll and J. M. Morel: Image denoising methods. A new nonlocal principle, SIAM Review 52 (2010), 113-147

[19] K. Burdzy and W. Werner: A counterexample to the "hot spots" conjecture, Ann. Math. 149 (1999), 309-317

[20] L. Caffarelli and L. Silvestre: Regularity theory for fully nonlinear integro-differential equations, Comm. Pure Appl. Math. 62 (2009), 597-638

[21] L. de Carli and S.M. Hudson: A Faber-Krahn inequality for solutions of Schrödinger's equation, Adv. Math. 230 (2012), 2416-2427

[22] R. Carmona, W.C. Masters and B. Simon: Relativistic Schrödinger operators: asymptotic behaviour of the eigenfunctions, J. Funct. Anal. 91 1990, 117-142

[23] Z.-Q. Chen and R. Song: Two-sided eigenvalue estimates for subordinate processes in domains, J. Funct. Anal. 226 (2005), no. 1, 90-113

[24] Z.-Q. Chen and R. Song: Continuity of eigenvalues of subordinate processes in domains, Math. Z. 252 (2006), 71-89

[25] I. Daubechies: An uncertainty principle for fermions with generalized kinetic energy, Commun. Math. Phys. 90 (1983), 319-334

[26] M. Donsker and S.R.S. Varadhan: On the principal eigenvalue of second-order elliptic differential operators, Comm. Pure Appl. Math. 29 (1976), no. 6, 595-621

[27] S.O. Durugo and J. Lörinczi: Spectral properties of the massless relativistic quartic oscillator, J. Differ. Equations 264 (2018), 3775-3809

[28] M. Fukushima, Y. Osima and M. Takeda: Dirichlet Forms and Symmetric Markov Processes, De Gruyter Studies in Mathematics 19, Walter de Gruyter, Berlin, Boston, 2010

[29] G. Gilboa and S. Osher: Nonlocal operators with applications to image processing, Multiscale Model. Simul. 7 (2009), 1005-1028

[30] R. Gorenflo, A.A. Kilbas, F. Mainardi and S.V. Rogosin: Mittag-Leffler Functions, Related Topics and Applications, Springer, 2014

[31] D. Grieser and D. Jerison: The size of the first eigenfunction of a convex planar domain $J$. Amer. Math. Soc. 11 (1998), no. 1, 41-72

[32] T. Grzywny: On Harnack inequality and Hölder regularity for isotropic unimodal Lévy processes, Potential Anal. 41 (2014), no. 1, 1-29

[33] P. Hartman and A. Wintner: On the infinitesimal generators of integral convolutions, Amer. J. Math. 64 (1942), 273-298

[34] A. Henrot, I. Lucardesi and G. Philippin: On two functionals involving the maximum of the torsion function, ESAIM COCV, doi.org/10.1051/cocv/2017069

[35] F. Hiroshima and J. Lőrinczi: Lieb-Thirring bound for Schrödinger operators with Bernstein functions of the Laplacian, Commun. Stoch. Anal. 6 (2012), no. 4, 589-602

[36] F. Hiroshima, T. Ichinose and J. Lörinczi: Path integral representation for Schrödinger operators with Bernstein functions of the Laplacian, Rev. Math. Phys. 24 (2012), no. 6, 1250013

[37] F. Hiroshima, T. Ichinose and J. Lörinczi: Probabilistic representation and fall-off of bound states of relativistic Schrödinger operators with spin 1/2, Publ. Res. Inst. Math. Sci. 49 (2013), 189-214

[38] N. Jacob: Pseudo-Differential Operators and Markov Processes, vols. 1-3, Imperial College Press, 2003-2005

[39] D. Jerison and N. Nadirashvili, The "hot spots" conjecture for domains with two axes of symmetry. J. Amer. Math. Soc. 13 (2000), no. 4, 741-772

[40] K. Kaleta, M. Kwaśnicki and J. Małecki: One-dimensional quasi-relativistic particle in the box, Rev. Math. Phys. 25 (2013), 1350014

[41] K. Kaleta, M. Kwaśnicki and J. Małecki: Asymptotic estimate of eigenvalues of pseudodifferential operators in an interval, J. Math. Anal. Appl. 439 (2016), 896-924 
[42] K. Kaleta and J. Lörinczi: Pointwise estimates of the eigenfunctions and intrinsic ultracontractivity-type properties of Feynman-Kac semigroups for a class of Lévy processes, Ann. Probab. 43 (2015), 1350-1398

[43] K. Kaleta and J. Lörinczi: Transition in the decay rates of stationary distributions of Lévy motion in an energy landscape, Phys. Rev. E 93 (2016), 022135

[44] K. Kaleta and J. Lörinczi: Fall-off of eigenfunctions of non-local Schrödinger operators with decaying potentials, Potential Anal. 46 (2017), 647-688

[45] J.F. Kelly and M.M. Meerschaert: The fractional advection-dispersion equation for contaminant transport, Ch.6 in: Handbook of Fractional Calculus with Applications, vol. 5 (V.E. Tarasov, ed.), Walter de Gruyter, 2019

[46] R. Klages, G. Radons and I.M. Sokolov: Anomalous Transport: Foundations and Applications, Wiley, 2008

[47] V. Knopova and R.L. Schilling: A note on the existence of transition probability densities of Lévy processes, Forum Math. 25 (2013), 125-149

[48] T. Kulczycki: On concavity of solutions of the Dirichlet problem for the equation $(-\Delta)^{1 / 2} \varphi=$ 1 in convex planar regions, J. Eur. Math. Soc. 19 (2017), 1361-1420

[49] T. Kulczycki, M. Kwaśnicki, J. Małecki and A. Stós: Spectral properties of the Cauchy process on half-line and interval, Proc. London Math. Soc. 101 (2010), 589-622

[50] M. Kwaśnicki: Spectral analysis of subordinate Brownian motions on the half-line, Studia Math 206 (2011), 211-271

[51] M. Kwaśnicki: Eigenvalues of the fractional Laplace operator in the interval, J. Funct. Anal. 262 (2012), 2379-2402

[52] T.L. Lai: Uniform Tauberian theorems and their applications to renewal theory and first passage problems, Ann. Probab. 4 (1976), no. 4, 628-643

[53] E.H. Lieb and R. Seiringer: The Stability of Matter in Quantum Mechanics, Cambridge University Press, 2010

[54] J. Lörinczi, unpublished notes, 2012

[55] J. Lőrinczi, F. Hiroshima, V. Betz: Feynman-Kac-Type Theorems and Gibbs Measures on Path Space. With Applications to Rigorous Quantum Field Theory, Walter de Gruyter, 2011; 2nd ed. forthcoming, 2019

[56] J. Lörinczi and J. Małecki: Spectral properties of the massless relativistic harmonic oscillator, J. Differ. Equations 253 (2012), 2846-2871

[57] J. Lörinczi and M. Ryznar: Local estimates of ground states of non-local Schrödinger operators, preprint, 2017

[58] M.M. Meerschaert and A. Sikorskii: Stochastic Models for Fractional Calculus, de Gruyter, 2011

[59] P.J. Méndez-Hernández: Brascamp-Lieb-Luttinger inequalities for convex domains of finite inradius, Duke Math. J. 113 (2002), 93-131

[60] S.T. Rachev and S. Mittnik: Stable Paretian Models in Finance, Wiley, 2000

[61] M. Rachh and S. Steinerberger: On the location of maxima of solutions of Schrödinger's equation, to appear in Comm. Pure. Appl. Math. 71 (2018), 1109-1122

[62] X. Ros-Oton and J. Serra: The Dirichlet problem for the fractional Laplacian: regularity up to the boundary, J. Math. Pures Appl. 101 (2014), 275-302

63] R.L. Schilling: Growth and Hölder conditions for the sample paths of Feller processes, Probab. Theory Relat. Fields 112 (1998), 565-611

[64] R. Schilling, R. Song, Z. Vondraček: Bernstein Functions, Walter de Gruyter, 2010

[65] A.S. Sznitman: Brownian Motion, Obstacles and Random Media, Springer, 1998

[66] M. van den Berg: Estimates for the torsion function and Sobolev constants. Potential Anal. 36 (2012), no. 4, 607-616 